\title{
Checklist of the parasites of European eel Anguilla anguilla (Linnaeus, 1758) (Anguillidae) in Poland
}

\author{
Joanna Dzido $\ddagger$, Leszek Rolbiecki ${ }^{\ddagger}$, Joanna N. Izdebska ${ }^{\ddagger}$, Rafał Bednarek ${ }^{\ddagger}$ \\ ‡ Department of Invertebrate Zoology and Parasitology, Faculty of Biology, University of Gdańsk, Wita Stwosza 59, Gdańsk, \\ Poland
}

Corresponding author: Leszek Rolbiecki (leszek.rolbiecki@ug.edu.pl)

Academic editor: Yasen Mutafchiev

Received: 23 Mar 2020 | Accepted: 28 May 2020 | Published: 10 Jun 2020

Citation: Dzido J, Rolbiecki L, Izdebska JN, Bednarek R (2020) Checklist of the parasites of European eel Anguilla anguilla (Linnaeus, 1758) (Anguillidae) in Poland. Biodiversity Data Journal 8: e52346. https://doi.org/10.3897/BDJ.8.e52346

\begin{abstract}
The present paper lists all parasite species of the European eel Anguilla anguilla (Linnaeus, 1758), recorded in Poland, in both its saltwater and freshwater habitats. The list has been drawn up, based on data acquired since 1844. The majority of included parasite species are presented with fish infection parameters together with data on their developmental stages and occupied microhabitats, localities and dates of collection of the eels themselves. The database includes 62 parasite taxa (including 50 species, nine identified to the genus level and three to higher taxa), representing at least 47 genera and 39 families. The most frequently-noted parasites of the European eel are the cestode Bothriocephalus claviceps, the nematodes Anguillicoloides crassus, Camallanus lacustris and Raphidascaris acus and the acanthocephalan Acanthocephalus lucii. Four alien species have been noted from this host: $A$. crassus, the monogeneans Pseudodactylogyrus anguillae and Pseudodactylogyrus bini and the acanthocephalan Paratenuisentis ambiguus. The present list includes both new host records and earlier records not included in previous lists of parasites of eels.
\end{abstract}

\section{Keywords}

biodiversity, eel, fish, parasite, species distribution 


\section{Introduction}

The European eel Anguilla anguilla (Linnaeus, 1758) is a species of fish with a wide distribution in European waters and one with both very high environmental and economic value. Therefore, there is a pressing need to understand the real and potential treats for eel populations, including such death hazards as parasitoses and their secondary consequences. Since the end of the 20th Century, eel populations have decreased by over $99 \%$ due to various factors, such as increased water pollution, climate change, overfishing and dam construction and the species is at risk of extinction. It is currently subject to protection by various forms of conservation, including the Washington Convention (CITES) and is listed as critically endangered by the IUCN Red List of Threatened Species (Dekker 2003, Stone 2003, Freyhof and Kottelat 2010). One significant factor in this decline was the appearance of the nematode Anguillicoloides crassus: an alien, invasive parasite which inhabits the swim bladders of eels, resulting in sickness and the disturbance of various vital functions that may prevent the eels from reaching their spawning area and reproducing (Moriarty and Dekker 1997, Lefebvre et al. 2002, Dekker 2003, Kirk 2003, Stone 2003, van Ginneken and Maes 2005). Anguillicoloides crassus was probably introduced from Taiwan, where it was associated with its specific host, the Japanese eel; since its introduction, it has spread rapidly throughout the European eel population (Taraschewski et al. 1987, Moravec 1992, Molnár et al. 1993, Münderle et al. 2006).

A comprehensive analysis of the European eel parasite fauna is complicated by its wide geographical distribution and by the poor understanding of the complex biology of the eel. It is therefore often difficult to draw firm conclusions on the infection routes with parasites or participation of the eel in their life cycles. Eel leptocephalus larvae migrate across the Atlantic Ocean to the coasts of Europe; then they metamorphose into glass eel (montée) and move to rivers and lakes, where they mature. Finally, they take part in catadromous migration to the spawning area in the Sargasso Sea (van Ginneken and Maes 2005). Thus, they inhabit different environmental conditions at different stages of ontogenesis; in addition, during the course of their migrations, they may accumulate parasites originating from different areas and further disseminate them. Parasite accumulation is also supported by their longevity and predatory lifestyle, the oldest known specimen in the wild being 85 years old (Dekker et al. 1998). The eel may constitute a significant link in the life cycles of parasites and their distribution in the environment; however, some differences may be attributed to local factors.

Therefore, there is a clear need to better understand the parasite fauna of European eels, its species composition, structural changes and infection level, both on the global and regional scales. Constant parasitological monitoring in all distribution areas would provide a clearer picture of the formation of parasite assemblages across the different parts of the distribution of the eel; it would also allow observation of changes in the parasite ranges and hence, any associated threats. However, being rather local in nature, data on the eel parasite fauna are scattered across a range of publications and reports; in addition, collective analyses are often further complicated by variations in sources or language barrier. Thus far, three checklists of eel parasites have been published concerning various 
species of Anguilla: two of them are from Japan, the second being a revised and updated checklist, while the other concerns the parasites found in $A$. anguilla in Europe and North Africa (Nagasawa et al. 2007, Jakob et al. 2016, Nagasawa and Hirotaka 2017). Out of necessity, the latter list is restricted to available sources from selected countries. Its aim was to provide an overview of the parasite fauna acting as the reference point to future analyses of trends in changes in biodiversity. However, this work did not provide a full picture of the data from Poland, as it included only seven original studies; in contrast, the present checklist includes 59.

Therefore, the objective of this study was to provide a complete, verified list of parasitic Protista and Metazoa found, thus far, in the European eel in Poland.

\section{Material and methods}

The checklist has been drawn up primarily on the basis of published data (55 items) from the area of Poland, including data from the period 1844-2016. It also includes our own unpublished data, marked in the table as "this study", together with examples of data from conference abstracts regarding the occurrence of $A$. crassus or the presence of new records from Poland.

For the majority of species, additional data have been provided if included in the source publications: infection parameters such as prevalence $(P)$, mean intensity, intensity range and abundance, as well as the developmental stages of the parasites and their microhabitats. The infection parameters were calculated, based on data included in original studies by means of unification, where possible. Information on dates of fish collection, as well as the geographical location (with GPS coordinates in the Suppl. material 1), have been also included.

The species were arranged in taxonomic and then alphabetical order. The Protista taxonomy follows Lom and Dyková (1992); for Trematoda, Gibson et al. (2002) and Jones et al. (2005); for Nematoda, Moravec (2013) and Nadler et al. (2005); for Cestoda, Kahlil et al. (1994); for Acanthocephala, Amin (2013); the taxonomy for Myxozoa, Monogenea, Arthropoda, Annelida and Mollusca follows the WORMS database (WoRMS Editorial Board 2020). As some taxa have been subject to revision over the years, valid and verified species names were used in the list. For instance, Szidat (1944) considered Sphaerostomum bramae (Müller, 1776), a trematode from the study of Markowski (1933) as Plagioporus angulatus. Furthermore, Spironucleus mobilis Wierzbicka \& EinszpornOrecka, 1986 is currently a synonym of Spironucleus anguillae (Lom and Dyková 1992), Trichophrya piscium Buetschli, 1889 is Capriniana piscium (Svobodová et al. 2009), Sphaerospora sphaerocapsularae Wierzbicka 1986 is now Ortholinea sphaerocapsularae (Sitjà-Bobadilla and Alvarez-Pellitero 1994), Sphaerospora anguillae Wierzbicka, 1986 is a synonym of S. gilsoni (Wierzbicka 1994), Ascaris labiata Rudolphi, 1809 is Raphidascaris acus, Anguillicola crassus Kuwahara, Niimi \& Itagaki, 1974 is currently known as Anguillicoloides crassus (Moravec 2006) and Contracaecum aduncum (Rudolphi, 1802) is Hysterothylacium aduncum. Bielecki et al. (2011) believe that Cystobranchus respirans 
(Troschel, 1850) is Piscicola respirans. In addition, it is known that "Diplostomum spathaceum" includes more than one species; there are several species of Diplostomum difficult to identify without using molecular methods (Niewiadomska 2003, Georgieva et al. 2013). Likewise, there is a possibility of wrong species identifitaciton in the case of Pomphorhynchus laevis, due to morphological similarity to $P$. tereticollis which occurs sympatrically (Špakulová et al. 2011, Hohenadler et al. 2018).

\section{Results and Discussion}

A total of 62 taxa have been recorded from the European eel in Poland, of which 11 are Protista (eight species and three identified at the genus level), including five Ciliophora (one identified at the genus level as Apiosoma) and two Apicomplexa. Of the 51 representatives of Metazoa (42 identified as species, six to genus level, three to higher taxa), six were Myxozoa, nine Trematoda, five Monogenea (one identified at the genus level - Dactylogyrus and one as Monogenea), five Cestoda (one identified as Cestoda gen. sp. Pseudophyllidearum larvae), ten Nematoda, eight Acanthocephala, two Annelida, three Arthropoda, one Mollusca (identified as Unionidae). In addition to the species mentioned in Table 1, further data on the occurrence of Acanthocephalus clavula and Corynosoma semerme in the European eel were given in overview studies on the parasites of the Polish ichthyofauna (Pojmańska et al. 2007, Popiołek 2016); however, this information was not included in the analysed original studies.

Table 1.

Checklist of the protozoan and metazoan parasites of Anguilla anguilla from Poland. The parameters have been provided with accuracy of original data; if the parameter was not given in the original work, it was marked with a dash (-). Parasite life stage: $A$ - adults, $e-$ eggs, $F-$ female, $L-$ larvae, $\mathrm{M}$ - male, met - metacercariae, $\mathrm{pA}$ - preadults, $\mathrm{pl}$ - plasmodia. Microhabitat: $\mathrm{sb}$ - swim bladder, ub - urinary bladder. Intensity: $(+)-$ single to not numerous, $(++)-$ rather numerous to numerous, $(+++)-$ very numerous, $(++++)-$ mass occurrence.

\begin{tabular}{|c|c|c|c|c|c|c|c|}
\hline $\begin{array}{l}\text { Parasite } \\
\text { life } \\
\text { stage }\end{array}$ & $\begin{array}{l}\text { Micro } \\
\text { habitat }\end{array}$ & $\begin{array}{l}\mathbf{P} \\
{[\%]}\end{array}$ & $\begin{array}{l}\text { Intensity } \\
\text { range } \\
\text { (mean) }\end{array}$ & $\begin{array}{l}\text { Abun } \\
\text { dance }\end{array}$ & Locality & $\begin{array}{l}\text { Material } \\
\text { collection } \\
\text { year }\end{array}$ & References \\
\hline \multicolumn{8}{|c|}{ METAMONADA } \\
\hline \multicolumn{8}{|c|}{ Family Hexamitidae } \\
\hline \multicolumn{8}{|c|}{ Spironucleus anguillae Einszporn-Orecka, 1979} \\
\hline- & 1 & - & 2 & - & Szczecin Lagoon & 1974 & $\begin{array}{l}\text { Einszporn- } \\
\text { Orecka } 1979\end{array}$ \\
\hline- & intestine & $1.9^{3}$ & $(+)-(++)$ & - & Szczecin Lagoon & $1982-83$ & $\begin{array}{l}\text { Wierzbicka and } \\
\text { Einszporn- } \\
\text { Orecka 1986, } \\
\text { Wierzbicka and } \\
\text { Orecka-Grabda } \\
1994\end{array}$ \\
\hline
\end{tabular}




\begin{tabular}{|c|c|c|c|c|c|c|c|}
\hline $\begin{array}{l}\text { Parasite } \\
\text { life } \\
\text { stage }\end{array}$ & $\begin{array}{l}\text { Micro } \\
\text { habitat }\end{array}$ & $\begin{array}{l}\mathbf{P} \\
{[\%]}\end{array}$ & $\begin{array}{l}\text { Intensity } \\
\text { range } \\
\text { (mean) }\end{array}$ & $\begin{array}{l}\text { Abun } \\
\text { dance }\end{array}$ & Locality & $\begin{array}{l}\text { Material } \\
\text { collection } \\
\text { year }\end{array}$ & References \\
\hline- & intestine & 4.2 & $(++)$ & - & Oder mouth & $1982-83$ & $\begin{array}{l}\text { Wierzbicka and } \\
\text { Einszporn- } \\
\text { Orecka 1986, } \\
\text { Wierzbicka and } \\
\text { Orecka-Grabda } \\
1994\end{array}$ \\
\hline \multicolumn{8}{|l|}{ EUGLENOZOA } \\
\hline \multicolumn{8}{|c|}{ Family Trypanosomatidae } \\
\hline \multicolumn{8}{|c|}{ Trypanosoma granulosum Laveran \& Mesnil, 1909} \\
\hline trypomastigote & blood & 24 & $(0.2-16.0)^{4}$ & - & Lake Siecino & $1970-73$ & $\begin{array}{l}\text { Orecka-Grabda } \\
\text { and Wierzbicka } \\
1996\end{array}$ \\
\hline trypomastigote & blood & 68 & $(2.2-16.2)^{4}$ & - & Lake Dąbie & $1970-73$ & $\begin{array}{l}\text { Orecka-Grabda } \\
\text { and Wierzbicka } \\
1996\end{array}$ \\
\hline- & blood & - & - & - & $\begin{array}{l}\text { Szczecin } \\
\text { Lagoon, Lake } \\
\text { Dąbie }\end{array}$ & $1970-73$ & $\begin{array}{l}\text { Orecka-Grabda } \\
1986\end{array}$ \\
\hline- & 5 & $46.9^{3}$ & $(+)-(++)$ & - & Szczecin Lagoon & $1982-83$ & $\begin{array}{l}\text { Wierzbicka and } \\
\text { Orecka-Grabda } \\
1994\end{array}$ \\
\hline- & 5 & 95.8 & $(+)-(++)$ & - & Oder mouth & $1982-83$ & $\begin{array}{l}\text { Wierzbicka and } \\
\text { Orecka-Grabda } \\
1994\end{array}$ \\
\hline- & - & $50-60$ & - & - & Lake Śniardwy & 1989 & $\begin{array}{l}\text { Własow et al. } \\
1991\end{array}$ \\
\hline- & - & 50 & - & - & Lake Mamry & 1990 & $\begin{array}{l}\text { Własow et al. } \\
1991\end{array}$ \\
\hline- & blood & 100 & variable & - & River Rega & 2001-02 & Rząd et al. 2007 \\
\hline \multicolumn{8}{|c|}{ Trypanosoma sp. } \\
\hline- & blood & 24 & - & - & Lake Ińsko & 1993 & $\begin{array}{l}\text { Rząd and } \\
\text { Pilecka-Rapacz } \\
2002\end{array}$ \\
\hline \multicolumn{8}{|l|}{ CILIOPHORA } \\
\hline \multicolumn{8}{|c|}{ Family Epistylididae } \\
\hline \multicolumn{8}{|l|}{ Apiosoma sp. } \\
\hline- & - & 6 & - & - & $\begin{array}{l}\text { Lake Dąbrowa } \\
\text { Wielka }\end{array}$ & $1990-91$ & $\begin{array}{l}\text { Własow et al. } \\
1991\end{array}$ \\
\hline \multicolumn{8}{|c|}{ Family Ichthyophthiriidae } \\
\hline \multicolumn{8}{|c|}{ Ichthyophthirius multifiliis (Fouquet, 1876) } \\
\hline- & gills, skin & - & - & - & River Darłówka & - & Grabda 1971 \\
\hline
\end{tabular}




\begin{tabular}{|c|c|c|c|c|c|c|c|}
\hline $\begin{array}{l}\text { Parasite } \\
\text { life } \\
\text { stage }\end{array}$ & $\begin{array}{l}\text { Micro } \\
\text { habitat }\end{array}$ & $\begin{array}{l}\mathbf{P} \\
{[\%]}\end{array}$ & $\begin{array}{l}\text { Intensity } \\
\text { range } \\
\text { (mean) }\end{array}$ & $\begin{array}{l}\text { Abun } \\
\text { dance }\end{array}$ & Locality & $\begin{array}{l}\text { Material } \\
\text { collection } \\
\text { year }\end{array}$ & References \\
\hline- & gills & $3.1^{3}$ & $(+)$ & - & Szczecin Lagoon & $1982-83$ & $\begin{array}{l}\text { Wierzbicka and } \\
\text { Orecka-Grabda } \\
1994\end{array}$ \\
\hline \multicolumn{8}{|c|}{ Family Trichodinidae } \\
\hline \multicolumn{8}{|c|}{ Trichodina jadranica Raabe, 1958} \\
\hline- & gills & $68.6^{3}$ & $(+)-(++++)$ & - & Szczecin Lagoon & $1982-83$ & $\begin{array}{l}\text { Wierzbicka and } \\
\text { Orecka-Grabda } \\
1994\end{array}$ \\
\hline- & gills & 66.7 & $(+)$ & - & Oder mouth & $1982-83$ & $\begin{array}{l}\text { Wierzbicka and } \\
\text { Orecka-Grabda } \\
1994\end{array}$ \\
\hline \multicolumn{8}{|c|}{ Trichodina sp. } \\
\hline- & - & 30 & - & - & Lake Niegocin & 1989 & $\begin{array}{l}\text { Własow et al. } \\
1991\end{array}$ \\
\hline- & - & 8 & - & - & Lake Mamry & 1990 & $\begin{array}{l}\text { Własow et al. } \\
1991\end{array}$ \\
\hline- & - & 6 & - & - & $\begin{array}{l}\text { Lake Dabrowa } \\
\text { Wielka }\end{array}$ & $1990-91$ & $\begin{array}{l}\text { Własow et al. } \\
1991\end{array}$ \\
\hline \multicolumn{8}{|c|}{ Trichodinella epizootica (Raabe, 1950) } \\
\hline- & gills & $62.9^{3}$ & $(+)-(+++)$ & - & Szczecin Lagoon & $1982-83$ & $\begin{array}{l}\text { Wierzbicka and } \\
\text { Orecka-Grabda } \\
1994\end{array}$ \\
\hline- & gills & 41.7 & $(+)-(+++)$ & - & Oder mouth & $1982-83$ & $\begin{array}{l}\text { Wierzbicka and } \\
\text { Orecka-Grabda } \\
1994\end{array}$ \\
\hline- & - & 3.3 & $1-4(2.5)$ & - & Vistula Lagoon & 2005 & $\begin{array}{l}\text { Rolbiecki and } \\
\text { Rokicki } 2006\end{array}$ \\
\hline \multicolumn{8}{|c|}{ Family Trichophryidae } \\
\hline \multicolumn{8}{|c|}{ Capriniana piscium (Bütschli, 1889) } \\
\hline- & gills & 4.2 & $(+++)$ & - & Oder mouth & $1982-83$ & $\begin{array}{l}\text { Wierzbicka and } \\
\text { Orecka-Grabda } \\
1994\end{array}$ \\
\hline \multicolumn{8}{|c|}{ APICOMPLEXA } \\
\hline \multicolumn{8}{|c|}{ Family Eimeriidae } \\
\hline \multicolumn{8}{|c|}{ Eimeria anguillae Léger \& Hollande, 1922} \\
\hline oocyst & intestine & $11.9^{3}$ & $(+)-(++++)$ & - & Szczecin Lagoon & $1982-83$ & $\begin{array}{l}\text { Wierzbicka and } \\
\text { Orecka-Grabda } \\
1994\end{array}$ \\
\hline oocyst & intestine & 20.8 & $(+)-(+++)$ & - & Oder mouth & $1982-83$ & $\begin{array}{l}\text { Wierzbicka and } \\
\text { Orecka-Grabda } \\
1994\end{array}$ \\
\hline
\end{tabular}




\begin{tabular}{|c|c|c|c|c|c|c|c|}
\hline $\begin{array}{l}\text { Parasite } \\
\text { life } \\
\text { stage }\end{array}$ & $\begin{array}{l}\text { Micro } \\
\text { habitat }\end{array}$ & $\begin{array}{l}\mathbf{P} \\
{[\%]}\end{array}$ & $\begin{array}{l}\text { Intensity } \\
\text { range } \\
\text { (mean) }\end{array}$ & $\begin{array}{l}\text { Abun } \\
\text { dance }\end{array}$ & Locality & $\begin{array}{l}\text { Material } \\
\text { collection } \\
\text { year }\end{array}$ & References \\
\hline \multicolumn{8}{|c|}{ Rhabdospora thelohani Laguessé, 1895} \\
\hline- & - & 20 & - & - & Lake Niegocin & 1989 & $\begin{array}{l}\text { Własow et al. } \\
1991\end{array}$ \\
\hline- & - & $50-60$ & - & - & Lake Śniardwy & 1989 & $\begin{array}{l}\text { Własow et al. } \\
1991\end{array}$ \\
\hline- & - & 17 & - & - & Lake Mamry & 1990 & $\begin{array}{l}\text { Własow et al. } \\
1991\end{array}$ \\
\hline- & - & 49 & - & - & $\begin{array}{l}\text { Lake Dąbrowa } \\
\text { Wielka }\end{array}$ & $1990-91$ & $\begin{array}{l}\text { Własow et al. } \\
1991\end{array}$ \\
\hline \multicolumn{8}{|l|}{ MYXOZOA } \\
\hline \multicolumn{8}{|c|}{ Family Myxidiidae } \\
\hline \multicolumn{8}{|c|}{ Myxidium giardi Cépède, 1906} \\
\hline spores & 7 & $79.9^{3}$ & $(+)-(+++)$ & - & Szczecin Lagoon & $1982-83$ & $\begin{array}{l}\text { Wierzbicka and } \\
\text { Orecka-Grabda } \\
1994\end{array}$ \\
\hline spores & 7 & 100 & $(+)-(++++)$ & - & Oder mouth & $1982-83$ & $\begin{array}{l}\text { Wierzbicka and } \\
\text { Orecka-Grabda } \\
1994\end{array}$ \\
\hline- & gills & 20 & $(+)-(++)$ & - & Lake Miedwie & $1997-99$ & $\begin{array}{l}\text { Sobecka and } \\
\text { Piasecki } 2002\end{array}$ \\
\hline- & - & 21.1 & $(++)-(+++)$ & - & Vistula Lagoon & 2005 & $\begin{array}{l}\text { Rolbiecki and } \\
\text { Rokicki } 2006\end{array}$ \\
\hline \multicolumn{8}{|c|}{ Zschokkella stettinensis Wierzbicka, 1987} \\
\hline spores & ub & 48.65 & $(+)-(+++)$ & - & $\begin{array}{l}\text { Szczecin } \\
\text { Lagoon, Lake } \\
\text { Dąbie }\end{array}$ & $\begin{array}{l}1983 \\
1985\end{array}$ & Wierzbicka 1987 \\
\hline- & ub & $11.3^{3}$ & $(+)-(+++)$ & - & Szczecin Lagoon & $1982-83$ & $\begin{array}{l}\text { Wierzbicka and } \\
\text { Orecka-Grabda } \\
1994\end{array}$ \\
\hline- & ub & 50.0 & $(+)-(+++)$ & - & Oder mouth & $1982-83$ & $\begin{array}{l}\text { Wierzbicka and } \\
\text { Orecka-Grabda } \\
1994\end{array}$ \\
\hline \multicolumn{8}{|c|}{ Family Myxobolidae } \\
\hline \multicolumn{8}{|c|}{ Henneguya psorospermica Thélohan, 1895} \\
\hline- & - & 6 & - & - & $\begin{array}{l}\text { Lake Dąbrowa } \\
\text { Wielka }\end{array}$ & $1990-91$ & $\begin{array}{l}\text { Własow et al. } \\
1991\end{array}$ \\
\hline \multicolumn{8}{|c|}{ Myxobolus portucalensis Saraiva \& Molnar, 1990} \\
\hline pl, spores & 8 & 16.9 & $(+)-(++)$ & - & Szczecin Lagoon & $1982-83$ & $\begin{array}{l}\text { Wierzbicka and } \\
\text { Orecka-Grabda } \\
1996\end{array}$ \\
\hline
\end{tabular}




\begin{tabular}{|c|c|c|c|c|c|c|c|}
\hline $\begin{array}{l}\text { Parasite } \\
\text { life } \\
\text { stage }\end{array}$ & $\begin{array}{l}\text { Micro } \\
\text { habitat }\end{array}$ & $\begin{array}{l}\mathbf{P} \\
{[\%]}\end{array}$ & $\begin{array}{l}\text { Intensity } \\
\text { range } \\
\text { (mean) }\end{array}$ & $\begin{array}{l}\text { Abun } \\
\text { dance }\end{array}$ & Locality & $\begin{array}{l}\text { Material } \\
\text { collection } \\
\text { year }\end{array}$ & References \\
\hline pl, spores & 8 & 29.2 & $(+)-(++)$ & - & Skolwiński Canal & $1982-83$ & $\begin{array}{l}\text { Wierzbicka and } \\
\text { Orecka-Grabda } \\
1996\end{array}$ \\
\hline pl, spores & 8 & 38.5 & $(+)-(++)$ & - & Lake Dąbie & 1985 & $\begin{array}{l}\text { Wierzbicka and } \\
\text { Orecka-Grabda } \\
1996\end{array}$ \\
\hline \multicolumn{8}{|c|}{ Family Ortholineidae } \\
\hline \multicolumn{8}{|c|}{ Ortholinea sphaerocapsularae (Wierzbicka, 1986) } \\
\hline spores & $u b$ & 7.69 & $(++)$ & - & Lake Dąbie & 1985 & $\begin{array}{l}\text { Wierzbicka } \\
\text { 1986b }\end{array}$ \\
\hline \multicolumn{8}{|c|}{ Family Sphaerosporidae } \\
\hline \multicolumn{8}{|c|}{ Sphaerospora gilsoni (Debaisieux, 1925) } \\
\hline pl, spores & ub & $13.2^{3}$ & $(+)-(+++)$ & - & Szczecin Lagoon & $1982-83$ & $\begin{array}{l}\text { Wierzbicka and } \\
\text { Orecka-Grabda } \\
1994\end{array}$ \\
\hline $\mathrm{pl}$, spores & ub & 87.5 & $(+)-(++++)$ & - & Oder mouth & $1982-83$ & $\begin{array}{l}\text { Wierzbicka and } \\
\text { Orecka-Grabda } \\
1994\end{array}$ \\
\hline spores & $u b$ & 87.5 & $(+)-(+++)$ & - & Szczecin Lagoon & 1983 & $\begin{array}{l}\text { Wierzbicka } \\
\text { 1986a }\end{array}$ \\
\hline \multicolumn{8}{|c|}{ PLATYHELMINTHES: TREMATODA } \\
\hline \multicolumn{8}{|c|}{ Family Allocreadiidae } \\
\hline \multicolumn{8}{|c|}{ Bunodera luciopercae (Müller, 1776) } \\
\hline A & - & 1.1 & 2 & 0.023 & Vistula Lagoon & 2005 & $\begin{array}{l}\text { Rolbiecki and } \\
\text { Rokicki } 2006\end{array}$ \\
\hline \multicolumn{8}{|c|}{ Family Azygiidae } \\
\hline \multicolumn{8}{|c|}{ Azygia lucii (Müller, 1776) } \\
\hline- & stomach & $0.63^{3}$ & $1(1)$ & $0.006^{3}$ & Szczecin Lagoon & $1982-83$ & $\begin{array}{l}\text { Orecka-Grabda } \\
\text { and Wierzbicka } \\
1994\end{array}$ \\
\hline \multicolumn{8}{|c|}{ Family Deropristidae } \\
\hline \multicolumn{8}{|c|}{ Deropristis inflata (Molin, 1859) } \\
\hline A & intestine & $18.5^{3}$ & $1-12\left(3.8^{3}\right)$ & $0.70^{3}$ & $\begin{array}{l}\text { Baltic Sea (n. } \\
\text { Chłapowo), Puck } \\
\text { Bay }\end{array}$ & 1930-31 & Markowski 1933 \\
\hline- & intestine & $17.6^{3}$ & $2-30(13)$ & - & Gulf of Gdańsk & $1967-71$ & Rokicki 1975 \\
\hline- & - & 3 & $1(1)$ & $0.03^{3}$ & Lake Dąbie & 1971 & Seyda 1973 \\
\hline- & intestine & $3.8^{3}$ & $1-63(14.83)$ & $0.56^{3}$ & Szczecin Lagoon & $1982-83$ & $\begin{array}{l}\text { Orecka-Grabda } \\
\text { and Wierzbicka } \\
1994\end{array}$ \\
\hline
\end{tabular}




\begin{tabular}{|c|c|c|c|c|c|c|c|}
\hline $\begin{array}{l}\text { Parasite } \\
\text { life } \\
\text { stage }\end{array}$ & $\begin{array}{l}\text { Micro } \\
\text { habitat }\end{array}$ & $\begin{array}{l}\mathbf{P} \\
{[\%]}\end{array}$ & $\begin{array}{l}\text { Intensity } \\
\text { range } \\
\text { (mean) }\end{array}$ & $\begin{array}{l}\text { Abun } \\
\text { dance }\end{array}$ & Locality & $\begin{array}{l}\text { Material } \\
\text { collection } \\
\text { year }\end{array}$ & References \\
\hline A & intestine & 1.43 & $2-4$ & 0.042 & Dead Vistula & $1982-90$ & $\begin{array}{l}\text { Sulgostowska } \\
1993\end{array}$ \\
\hline A & intestine & 12.17 & $1-600$ & 2.190 & Gulf of Gdańsk & $1982-90$ & $\begin{array}{l}\text { Sulgostowska } \\
1993\end{array}$ \\
\hline A & intestine & 42.15 & $1-200$ & 16.016 & $\begin{array}{l}\text { Baltic Sea (n. } \\
\text { Władysławowo) }\end{array}$ & $1982-90$ & $\begin{array}{l}\text { Sulgostowska } \\
1993\end{array}$ \\
\hline- & intestine & 1.14 & $2(2.00)$ & 0.02 & Lake Łebsko & $2000-06$ & $\begin{array}{l}\text { Morozińska- } \\
\text { Gogol } 2011\end{array}$ \\
\hline- & - & 0.4 & $1(1)$ & $0.002^{3}$ & Vistula Lagoon & 2001-02 & $\begin{array}{l}\text { Bystydzieńska et } \\
\text { al. } 2005\end{array}$ \\
\hline- & - & 0.7 & $1(1)$ & $0.007^{3}$ & Puck Bay & 2002 & $\begin{array}{l}\text { Bystydzieńska et } \\
\text { al. } 2005\end{array}$ \\
\hline A & - & 2.2 & $2-6(3)$ & $0.09^{3}$ & Vistula Lagoon & 2005 & $\begin{array}{l}\text { Rolbiecki and } \\
\text { Rokicki } 2006\end{array}$ \\
\hline \multicolumn{8}{|c|}{ Family Diplostomidae } \\
\hline \multicolumn{8}{|c|}{ Diplostomum spathaceum s. I. (Rudolphi, 1819) } \\
\hline met & - & 10 & $1(1)$ & $0.10^{3}$ & $\begin{array}{l}\text { Oder (n. } \\
\text { Stołczyn) }\end{array}$ & 1971 & Seyda 1973 \\
\hline met & - & 31 & $1-4\left(1.6^{3}\right)$ & $0.50^{3}$ & Szczecin Lagoon & 1971 & Seyda 1973 \\
\hline met & - & 6 & $1(1)$ & $0.10^{3}$ & Lake Dąbie & 1971 & Seyda 1973 \\
\hline \multicolumn{8}{|c|}{ Diplostomum spp. } \\
\hline met & - & 9.1 & $2^{3}\left(2.0^{3}\right)$ & 0.2 & Lake Dgał Wielki & $1979-84$ & $\begin{array}{l}\text { Grabda- } \\
\text { Kazubska et al. } \\
1987\end{array}$ \\
\hline met & - & 6 & - & - & Lake Mamry & 1990 & $\begin{array}{l}\text { Własow et al. } \\
1991\end{array}$ \\
\hline met & - & 19 & - & - & $\begin{array}{l}\text { Lake Dabrowa } \\
\text { Wielka }\end{array}$ & $1990-91$ & $\begin{array}{l}\text { Własow et al. } \\
1991\end{array}$ \\
\hline met & eyes & 3.41 & $1-3(3.67)$ & 0.13 & Lake Łebsko & 2000-06 & $\begin{array}{l}\text { Morozińska- } \\
\text { Gogol 2007, } \\
\text { Morozińska- } \\
\text { Gogol } 2011\end{array}$ \\
\hline met & eyes & 51.1 & $1-88(6.8)$ & - & Puck Bay & 2002 & $\begin{array}{l}\text { Bystydzieńska et } \\
\text { al. } 2005\end{array}$ \\
\hline met & - & 8.9 & $1-2(1.4)$ & - & Vistula Lagoon & 2005 & $\begin{array}{l}\text { Rolbiecki and } \\
\text { Rokicki } 2006\end{array}$ \\
\hline met & eye lens & $14.3^{9}$ & $6(6.0)$ & 0.85 & Rzeka Łeba & 2014-15 & This study \\
\hline \multicolumn{8}{|c|}{ Tylodelphys clavata (Nordmann, 1832) } \\
\hline met & $\begin{array}{l}\text { vitreous } \\
\text { humour }\end{array}$ & 3 & $1(1)$ & $0.03^{3}$ & Szczecin Lagoon & 1971 & Seyda 1973 \\
\hline
\end{tabular}




\begin{tabular}{|c|c|c|c|c|c|c|c|}
\hline $\begin{array}{l}\text { Parasite } \\
\text { life } \\
\text { stage }\end{array}$ & $\begin{array}{l}\text { Micro } \\
\text { habitat }\end{array}$ & $\begin{array}{l}\mathbf{P} \\
{[\%]}\end{array}$ & $\begin{array}{l}\text { Intensity } \\
\text { range } \\
\text { (mean) }\end{array}$ & $\begin{array}{l}\text { Abun } \\
\text { dance }\end{array}$ & Locality & $\begin{array}{l}\text { Material } \\
\text { collection } \\
\text { year }\end{array}$ & References \\
\hline met & $\begin{array}{l}\text { vitreous } \\
\text { humour }\end{array}$ & 3 & $1(1)$ & $0.03^{3}$ & Lake Dabie & 1971 & Seyda 1973 \\
\hline \multicolumn{8}{|c|}{ Family Hemiuridae } \\
\hline \multicolumn{8}{|c|}{ Brachyphallus crenatus (Rudolphi, 1802) } \\
\hline- & - & $5.9^{3}$ & 9 & $0.53^{3}$ & Gulf of Gdańsk & $1967-71$ & Rokicki 1975 \\
\hline- & - & 0.2 & $1(1)$ & $0.002^{3}$ & Vistula Lagoon & 2001-02 & $\begin{array}{l}\text { Bystydzieńska et } \\
\text { al. } 2005\end{array}$ \\
\hline \multicolumn{8}{|c|}{ Family Opecoelidae } \\
\hline \multicolumn{8}{|c|}{ Plagioporus angulatus (Dujardin, 1845) } \\
\hline A & intestine & $7.4^{3}$ & $1-3(2)$ & $0.15^{3}$ & $\begin{array}{l}\text { Baltic Sea (n. } \\
\text { Chłapowo) }\end{array}$ & $1930-31$ & Markowski 1933 \\
\hline \multicolumn{8}{|c|}{ Family Strigeidae } \\
\hline \multicolumn{8}{|c|}{ Ichthyocotylurus platycephalus (Creplin, 1825) } \\
\hline met & stomach ${ }^{10}$ & $0.63^{3}$ & $3(3)$ & $0.02^{3}$ & Szczecin Lagoon & $1982-83$ & $\begin{array}{l}\text { Orecka-Grabda } \\
\text { and Wierzbicka } \\
1994\end{array}$ \\
\hline met & - & 2.2 & $3(3)$ & $0.07^{3}$ & Vistula Lagoon & 2005 & $\begin{array}{l}\text { Rolbiecki and } \\
\text { Rokicki } 2006\end{array}$ \\
\hline \multicolumn{8}{|c|}{ PLATYHELMINTHES: MONOGENEA } \\
\hline \multicolumn{8}{|c|}{ Family Dactylogyridea } \\
\hline \multicolumn{8}{|c|}{ Dactylogyrus sp. } \\
\hline- & gills & 30 & (1) & - & Lake Miedwie & $1997-99$ & $\begin{array}{l}\text { Sobecka and } \\
\text { Piasecki } 2002\end{array}$ \\
\hline \multicolumn{8}{|c|}{ Family Pseudodactylogyridae } \\
\hline \multicolumn{8}{|c|}{ Pseudodactylogyrus anguillae (Yin \& Sproston, 1948) } \\
\hline- & gills & - & - & $3.46^{3}$ & Lake Strażyn & - & Dzika et al. 1995 \\
\hline- & gills & 90 & $(11.6)$ & 10.4 & Lake Dębno & 1994-95 & Dzika 1999 \\
\hline- & gills & 11 & $(1.82-9.25)$ & $0.14-4.78$ & $\begin{array}{l}\text { Rivers Radew, } \\
\text { Rega, Wieprza }\end{array}$ & $1999-2003$ & $\begin{array}{l}\text { Sobecka and } \\
\text { Pilecka-Rapacz } \\
2003\end{array}$ \\
\hline- & gills & 1.13 & $3(3.0)$ & 0.03 & Lake Łebsko & $2000-06$ & $\begin{array}{l}\text { Morozińska- } \\
\text { Gogol 2009, } \\
\text { Morozińska- } \\
\text { Gogol } 2011\end{array}$ \\
\hline- & gills & $100^{9}$ & $6-16(11)$ & $11.0^{3}$ & Puck Bay & 2002 & $\begin{array}{l}\text { Bystydzieńska et } \\
\text { al. } 2005\end{array}$ \\
\hline \multicolumn{8}{|c|}{ Pseudodactylogyrus bini Kikuchi, 1929} \\
\hline- & gills & - & - & $2.07^{3}$ & Lake Strażyn & - & Dzika et al. 1995 \\
\hline- & gills & 71 & $(18.1)$ & 16.2 & Lake Dębno & 1994-95 & Dzika 1999 \\
\hline
\end{tabular}




\begin{tabular}{|c|c|c|c|c|c|c|c|}
\hline $\begin{array}{l}\text { Parasite } \\
\text { life } \\
\text { stage }\end{array}$ & $\begin{array}{l}\text { Micro } \\
\text { habitat }\end{array}$ & $\begin{array}{l}\mathbf{P} \\
{[\%]}\end{array}$ & $\begin{array}{l}\text { Intensity } \\
\text { range } \\
\text { (mean) }\end{array}$ & $\begin{array}{l}\text { Abun } \\
\text { dance }\end{array}$ & Locality & $\begin{array}{l}\text { Material } \\
\text { collection } \\
\text { year }\end{array}$ & References \\
\hline- & gills & 11 & $(0.73-3.5)$ & $0.12-1.95$ & $\begin{array}{l}\text { River Radew, } \\
\text { Rega, Wieprza }\end{array}$ & $1999-2003$ & $\begin{array}{l}\text { Sobecka and } \\
\text { Pilecka-Rapacz } \\
2003\end{array}$ \\
\hline \multicolumn{8}{|c|}{ Pseudodactylogyrus sp. } \\
\hline- & gills & $1.9^{3}$ & $1(1)$ & $0.02^{3}$ & Szczecin Lagoon & $1982-83$ & $\begin{array}{l}\text { Orecka-Grabda } \\
\text { and Wierzbicka } \\
1994\end{array}$ \\
\hline \multicolumn{8}{|c|}{ Monogenea $\mathrm{n}$. det. } \\
\hline- & - & 17 & - & - & $\begin{array}{l}\text { Lake Dąbrowa } \\
\text { Wielka }\end{array}$ & $1990-91$ & $\begin{array}{l}\text { Własow et al. } \\
1991\end{array}$ \\
\hline \multicolumn{8}{|c|}{ PLATYHELMINTHES: CESTODA } \\
\hline \multicolumn{8}{|c|}{ Family Bothriocephalidae } \\
\hline \multicolumn{8}{|c|}{ Bothriocephalus claviceps (Goeze, 1782) } \\
\hline A & intestine & $22.2^{3}$ & $2-3\left(2.5^{3}\right)$ & $0.55^{3}$ & $\begin{array}{l}\text { Baltic Sea (n. } \\
\text { Chłapowo), Puck } \\
\text { Bay }\end{array}$ & 1930-31 & Markowski 1933 \\
\hline A & - & - & - & - & $\begin{array}{l}\text { Lake Gołdapiwo, } \\
\text { Lake Mamry }\end{array}$ & $1954-58$ & Jarecka 1959 \\
\hline A & intestine & $8.3^{3}$ & $1(1)$ & $0.08^{3}$ & Puck Bay & 1959 & Sołtyńska 1964 \\
\hline- & - & 25 & $1-11\left(4.2^{3}\right)$ & $1.05^{3}$ & $\begin{array}{l}\text { Oder (n. } \\
\text { Stołczyn) }\end{array}$ & 1971 & Seyda 1973 \\
\hline- & - & 37 & $1-14\left(4.9^{3}\right)$ & $1.84^{3}$ & Szczecin Lagoon & 1971 & Seyda 1973 \\
\hline- & - & 23 & $2-13\left(3.7^{3}\right)$ & $0.84^{3}$ & Lake Dąbie & 1971 & Seyda 1973 \\
\hline- & - & 9.1 & $3^{3}\left(3^{3}\right)$ & 0.27 & Lake Dgał Wielki & $1979-84$ & $\begin{array}{l}\text { Grabda- } \\
\text { Kazubska et al. } \\
1987\end{array}$ \\
\hline- & intestine & $35.2^{3}$ & $1-5\left(1.1^{3}\right)$ & $0.40^{3}$ & Szczecin Lagoon & $1982-83$ & $\begin{array}{l}\text { Orecka-Grabda } \\
\text { and Wierzbicka } \\
1994\end{array}$ \\
\hline- & intestine & 29.2 & $1-3\left(1.4^{3}\right)$ & $0.42^{3}$ & Skolwiński Canal & $1982-83$ & $\begin{array}{l}\text { Orecka-Grabda } \\
\text { and Wierzbicka } \\
1994\end{array}$ \\
\hline immature, $A$ & intestine & 10.00 & $1-35$ & 0.807 & Dead Vistula & $1982-90$ & $\begin{array}{l}\text { Sulgostowska } \\
1993\end{array}$ \\
\hline immature, $\mathrm{A}$ & intestine & 8.36 & $1-10$ & 0.183 & Gulf of Gdańsk & $1982-90$ & $\begin{array}{l}\text { Sulgostowska } \\
1993\end{array}$ \\
\hline immature, $A$ & intestine & 11.57 & $1-18$ & 0.371 & $\begin{array}{l}\text { Baltic Sea (n. } \\
\text { Władysławowo) }\end{array}$ & $1982-90$ & $\begin{array}{l}\text { Sulgostowska } \\
1993\end{array}$ \\
\hline- & - & 6 & - & - & $\begin{array}{l}\text { Lake Dabrowa } \\
\text { Wielka }\end{array}$ & $1990-91$ & $\begin{array}{l}\text { Własow et al. } \\
1991\end{array}$ \\
\hline- & intestine & 12.5 & $1-6(2.27)$ & 0.28 & Lake Łebsko & $2000-06$ & $\begin{array}{l}\text { Morozińska- } \\
\text { Gogol } 2011\end{array}$ \\
\hline
\end{tabular}




\begin{tabular}{|c|c|c|c|c|c|c|c|}
\hline $\begin{array}{l}\text { Parasite } \\
\text { life } \\
\text { stage }\end{array}$ & $\begin{array}{l}\text { Micro } \\
\text { habitat }\end{array}$ & $\begin{array}{l}\mathbf{P} \\
{[\%]}\end{array}$ & $\begin{array}{l}\text { Intensity } \\
\text { range } \\
\text { (mean) }\end{array}$ & $\begin{array}{l}\text { Abun } \\
\text { dance }\end{array}$ & Locality & $\begin{array}{l}\text { Material } \\
\text { collection } \\
\text { year }\end{array}$ & References \\
\hline- & - & 0.8 & $1-2(1.5)$ & - & Vistula Lagoon & 2001-02 & $\begin{array}{l}\text { Bystydzieńska et } \\
\text { al. } 2005\end{array}$ \\
\hline- & - & 30.1 & $1-11(2.8)$ & - & Puck Bay & 2002 & $\begin{array}{l}\text { Bystydzieńska et } \\
\text { al. } 2005\end{array}$ \\
\hline A & intestine & 15.2 & $1-14(3.4)$ & 0.52 & Lake Wdzydze & 2004 & This study \\
\hline A & - & 18.9 & $1-3(1.3)$ & - & Vistula Lagoon & 2005 & $\begin{array}{l}\text { Rolbiecki and } \\
\text { Rokicki } 2006\end{array}$ \\
\hline \multicolumn{8}{|c|}{ Family Proteocephalidae } \\
\hline \multicolumn{8}{|c|}{ Proteocephalus macrocephalus (Creplin, 1825) } \\
\hline A & intestine & $22.2^{3}$ & $1-20\left(5.0^{3}\right)$ & $1.10^{3}$ & $\begin{array}{l}\text { Baltic Sea (n. } \\
\text { Chłapowo), Puck } \\
\text { Bay }\end{array}$ & 1930-31 & Markowski 1933 \\
\hline plerocercoid & intestine & $8.3^{3}$ & $1(1)$ & $0.08^{3}$ & Puck Bay & 1959 & Sołtyńska 1964 \\
\hline- & intestine & $4.1^{3}$ & $1-19$ & - & Gulf of Gdańsk & $1967-71$ & Rokicki 1975 \\
\hline- & - & 10 & $1-3\left(2.0^{3}\right)$ & $0.20^{3}$ & $\begin{array}{l}\text { Oder (n. } \\
\text { Stołczyn) }\end{array}$ & 1971 & Seyda 1973 \\
\hline- & - & 22 & $1-10\left(2.9^{3}\right)$ & $0.62^{3}$ & Szczecin Lagoon & 1971 & Seyda 1973 \\
\hline- & - & 26 & $1-4\left(4.5^{3}\right)$ & $1.16^{3}$ & Lake Dąbie & 1971 & Seyda 1973 \\
\hline- & intestine & $39.0^{3}$ & $1-33\left(3.3^{3}\right)$ & $1.30^{3}$ & Szczecin Lagoon & $1982-83$ & $\begin{array}{l}\text { Orecka-Grabda } \\
\text { and Wierzbicka } \\
1994\end{array}$ \\
\hline- & intestine & 16.7 & $1-2\left(1.5^{3}\right)$ & $1.50^{3}$ & Skolwiński Canal & $1982-83$ & $\begin{array}{l}\text { Orecka-Grabda } \\
\text { and Wierzbicka } \\
1994\end{array}$ \\
\hline immature, $\mathrm{A}$ & intestine & 15.71 & $1-5$ & 0.300 & Dead Vistula & $1982-90$ & $\begin{array}{l}\text { Sulgostowska } \\
1993\end{array}$ \\
\hline immature, $A$ & intestine & 10.12 & $1-25$ & 0.253 & Gulf of Gdańsk & $1982-90$ & $\begin{array}{l}\text { Sulgostowska } \\
1993\end{array}$ \\
\hline immature, $A$ & intestine & 24.79 & $1-12$ & 0.743 & $\begin{array}{l}\text { Baltic Sea (n. } \\
\text { Władysławowo) }\end{array}$ & $1982-90$ & $\begin{array}{l}\text { Sulgostowska } \\
1993\end{array}$ \\
\hline- & intestine & 27.27 & $1-15(3.75)$ & 1.02 & Lake Łebsko & $2000-06$ & $\begin{array}{l}\text { Morozińska- } \\
\text { Gogol } 2011\end{array}$ \\
\hline- & - & 8.4 & $1-22(3.8)$ & - & Vistula Lagoon & 2001-02 & $\begin{array}{l}\text { Bystydzieńska et } \\
\text { al. } 2005\end{array}$ \\
\hline- & - & 23.3 & $1-42(4.8)$ & - & Puck Bay & 2002 & $\begin{array}{l}\text { Bystydzieńska et } \\
\text { al. } 2005\end{array}$ \\
\hline A & - & 31.1 & $1-5(2.5)$ & - & Vistula Lagoon & 2005 & $\begin{array}{l}\text { Rolbiecki and } \\
\text { Rokicki } 2006\end{array}$ \\
\hline$A$ & intestine & $20.0^{9}$ & $2(2.0)$ & 0.40 & River Szkarpawa & $2014-16$ & This study \\
\hline
\end{tabular}




\begin{tabular}{|c|c|c|c|c|c|c|c|}
\hline $\begin{array}{l}\text { Parasite } \\
\text { life } \\
\text { stage }\end{array}$ & $\begin{array}{l}\text { Micro } \\
\text { habitat }\end{array}$ & $\begin{array}{l}\mathbf{P} \\
{[\%]}\end{array}$ & $\begin{array}{l}\text { Intensity } \\
\text { range } \\
\text { (mean) }\end{array}$ & $\begin{array}{l}\text { Abun } \\
\text { dance }\end{array}$ & Locality & $\begin{array}{l}\text { Material } \\
\text { collection } \\
\text { year }\end{array}$ & References \\
\hline juvenile & - & 16.7 & $1-6(2.2)$ & - & Vistula Lagoon & 2005 & $\begin{array}{l}\text { Rolbiecki and } \\
\text { Rokicki } 2006\end{array}$ \\
\hline \multicolumn{8}{|c|}{ Family Triaenophoridae } \\
\hline \multicolumn{8}{|c|}{ Triaenophorus nodulosus (Pallas, 1781) } \\
\hline- & intestine & $20.0^{3,9}$ & 1 & 0.203 & $\begin{array}{l}\text { Vistula (n. } \\
\text { Warszawa) }\end{array}$ & $1924-25$ & Dabrowska 1970 \\
\hline \multicolumn{8}{|l|}{ Family n. det. } \\
\hline \multicolumn{8}{|c|}{ Cestoda gen. sp. Pseudophyllidarum larvae } \\
\hline L & intestine & $8.3^{3}$ & $1(1)$ & $0.08^{3}$ & Puck Bay & 1959 & Sołtyńska 1964 \\
\hline \multicolumn{8}{|l|}{ NEMATODA } \\
\hline \multicolumn{8}{|c|}{ Family Anguillicolidae } \\
\hline \multicolumn{8}{|c|}{ Anguillicoloides crassus (Kuwahara, Niimi \& Itagaki, 1974) } \\
\hline- & $\mathrm{sb}$ & 75 & - & - & Vistula Lagoon & 1988 & Grawiński 1994 \\
\hline- & $\mathrm{sb}$ & 80 & - & - & $\begin{array}{l}\text { Lakes } \\
\text { Przywłoczne, } \\
\text { Skape, } \\
\text { Wielewickie }\end{array}$ & 1989 & Grawiński 1994 \\
\hline- & - & 68.3 & $1-25$ & - & Vistula Lagoon & $1988-90$ & $\begin{array}{l}\text { Rolbiecki et al. } \\
1996\end{array}$ \\
\hline- & - & $2.7^{3}$ & $1-2$ & - & Lake Niegocin & 1989 & $\begin{array}{l}\text { Własow et al. } \\
1991\end{array}$ \\
\hline- & - & $70.0^{3}$ & $5-25$ & - & $\begin{array}{l}\text { Goczałkowicki } \\
\text { Reservoir }\end{array}$ & $1989-90$ & $\begin{array}{l}\text { Własow et al. } \\
1991\end{array}$ \\
\hline- & - & $2.7^{3}$ & $2-33$ & - & Lake Mamry & 1990 & $\begin{array}{l}\text { Własow et al. } \\
1991\end{array}$ \\
\hline- & - & - & - & - & 12 & - & Własow 1991 \\
\hline L2-L4, pA, A & $\mathrm{sb}$ & 78.3 & $1-204600$ & - & Lake Strażyn & 1993 & $\begin{array}{l}\text { Własow et al. } \\
1991\end{array}$ \\
\hline L2-L4, pA, A & $\mathrm{sb}$ & 25 & $1-102$ & - & $\begin{array}{l}\text { reservoir near } \\
\text { the village of Gaj }\end{array}$ & 1993 & $\begin{array}{l}\text { Własow et al. } \\
1991\end{array}$ \\
\hline- & - & 100 & $15-20$ & - & Vistula Lagoon & 1993 & Grawiński 1994 \\
\hline$L, A$ & $\mathrm{sb}$ & 78.7 & $1-15$ & - & Lake Ińsko & 1993 & $\begin{array}{l}\text { Orecka et al. } \\
1995\end{array}$ \\
\hline- & $\mathrm{sb}$ & 88.7 & $1-15$ & - & Lake Ińsko & 1993 & $\begin{array}{l}\text { Rząd and } \\
\text { Pilecka-Rapacz } \\
2002\end{array}$ \\
\hline juvenile, F & $\mathrm{sb}$ & $23.4^{3}$ & $0-36$ & - & Szczecin Lagoon & 1993-94 & $\begin{array}{l}\text { Garbacik- } \\
\text { Wesołowska et } \\
\text { al. } 1994\end{array}$ \\
\hline
\end{tabular}




\begin{tabular}{|c|c|c|c|c|c|c|c|}
\hline $\begin{array}{l}\text { Parasite } \\
\text { life } \\
\text { stage }\end{array}$ & $\begin{array}{l}\text { Micro } \\
\text { habitat }\end{array}$ & $\begin{array}{l}\mathbf{P} \\
{[\%]}\end{array}$ & $\begin{array}{l}\text { Intensity } \\
\text { range } \\
\text { (mean) }\end{array}$ & $\begin{array}{l}\text { Abun } \\
\text { dance }\end{array}$ & Locality & $\begin{array}{l}\text { Material } \\
\text { collection } \\
\text { year }\end{array}$ & References \\
\hline juvenile, F & $\mathrm{sb}$ & 69.0 & $0-22$ & - & Lake Łętowskie & 1994 & $\begin{array}{l}\text { Garbacik- } \\
\text { Wesołowska et } \\
\text { al. } 1994\end{array}$ \\
\hline juvenile, F & $\mathrm{sb}$ & $23.1^{3}$ & $0-10$ & - & Pomeranian Bay & 1994 & $\begin{array}{l}\text { Garbacik- } \\
\text { Wesołowska et } \\
\text { al. } 1994\end{array}$ \\
\hline L & $s b$ & 70 & $1-35$ & - & Szczecin Lagoon & $1994-96$ & $\begin{array}{l}\text { Rząd and } \\
\text { Pilecka-Rapacz } \\
2001\end{array}$ \\
\hline- & $s b$ & $37.5^{9}$ & $3-8(5.0)$ & - & Dead Vistula & 1996 & $\begin{array}{l}\text { Rolbiecki and } \\
\text { Rokicki } 2005\end{array}$ \\
\hline- & $s b$ & 37.5 & $5-16(7.3)$ & - & Lake Druzno & 1997 & $\begin{array}{l}\text { Rolbiecki and } \\
\text { Rokicki } 2005\end{array}$ \\
\hline$e, L 4, p A, A$ & $\begin{array}{l}\text { sb, } \\
\text { intestine }\end{array}$ & 41.9 & $(3.0)$ & - & Gulf of Gdańsk & $1997-98$ & $\begin{array}{l}\text { Rolbiecki et al. } \\
2000\end{array}$ \\
\hline- & $s b$ & 100 & $3-44(8.2)$ & 6.7 & Lake Miedwie & $1997-99$ & $\begin{array}{l}\text { Sobecka and } \\
\text { Piasecki } 2002\end{array}$ \\
\hline $\mathrm{L} 3, \mathrm{~L} 4, \mathrm{~A}$ & $\mathrm{sb}$ & 33.3 & $1-7$ & - & $\begin{array}{l}\text { River Wieprza } \\
\text { (near Darłowo) }\end{array}$ & 1999 & $\begin{array}{l}\text { Pilecka-Rapacz } \\
2001\end{array}$ \\
\hline $\mathrm{L} 3, \mathrm{~L} 4, \mathrm{~A}$ & $\mathrm{sb}$ & 40 & $1-10$ & - & $\begin{array}{l}\text { River Rega (near } \\
\text { Trzebiatów) }\end{array}$ & 1999 & $\begin{array}{l}\text { Pilecka-Rapacz } \\
2001\end{array}$ \\
\hline- & $\mathrm{sb}$ & 59.1 & $1-11(1.7)$ & - & $\begin{array}{l}\text { River Rega } \\
\text { (Lake Rejowice) }\end{array}$ & $1999-2003$ & $\begin{array}{l}\text { Pilecka-Rapacz } \\
\text { and Sobecka } \\
2004\end{array}$ \\
\hline- & $\mathrm{sb}$ & 41.7 & $1-8(1.3)$ & - & $\begin{array}{l}\text { River Wieprza } \\
\text { (nearDarłowo) }\end{array}$ & $\begin{array}{l}1999 \\
2001\end{array}$ & $\begin{array}{l}\text { Pilecka-Rapacz } \\
\text { and Sobecka } \\
2004\end{array}$ \\
\hline- & $s b$ & 65.6 & $1-12(2.1)$ & - & River Radew & $2000-01$ & $\begin{array}{l}\text { Pilecka-Rapacz } \\
\text { and Sobecka } \\
2004\end{array}$ \\
\hline$A, L$ & $\mathrm{sb}$ & 68.18 & $1-27(5.82)$ & 3.97 & Lake Łebsko & $2000-06$ & $\begin{array}{l}\text { Morozińska- } \\
\text { Gogol 2005, } \\
\text { Morozińska- } \\
\text { Gogol 2007, } \\
\text { Morozińska- } \\
\text { Gogol 2009, } \\
\text { Morozińska- } \\
\text { Gogol } 2011\end{array}$ \\
\hline- & $\mathrm{sb}$ & 66.7 & $11-24(17.5)$ & - & Lake Bukowo & $2000-07$ & $\begin{array}{l}\text { Morozińska- } \\
\text { Gogol } 2009\end{array}$ \\
\hline \multirow[t]{2}{*}{-} & $\mathrm{sb}$ & 100 & $5-11(8.0)$ & - & Lake Kopań & $2000-07$ & $\begin{array}{l}\text { Morozińska- } \\
\text { Gogol } 2009\end{array}$ \\
\hline & - & 75 & $1-58(10)$ & - & Vistula Lagoon & 2001-02 & $\begin{array}{l}\text { Bystydzieńska et } \\
\text { al. } 2005\end{array}$ \\
\hline L4, L5, A & $\mathrm{sb}$ & $75.9^{3}$ & $1-11(3.2)$ & - & River Rega & 2001-02 & Rząd et al. 2007 \\
\hline
\end{tabular}




\begin{tabular}{|c|c|c|c|c|c|c|c|}
\hline $\begin{array}{l}\text { Parasite } \\
\text { life } \\
\text { stage }\end{array}$ & $\begin{array}{l}\text { Micro } \\
\text { habitat }\end{array}$ & $\begin{array}{l}\mathbf{P} \\
{[\%]}\end{array}$ & $\begin{array}{l}\text { Intensity } \\
\text { range } \\
\text { (mean) }\end{array}$ & $\begin{array}{l}\text { Abun } \\
\text { dance }\end{array}$ & Locality & $\begin{array}{l}\text { Material } \\
\text { collection } \\
\text { year }\end{array}$ & References \\
\hline- & - & 74.4 & $1-62(8.3)$ & - & Puck Bay & 2002 & $\begin{array}{l}\text { Bystydzieńska et } \\
\text { al. } 2005\end{array}$ \\
\hline L2-L4, A, e & $\mathrm{sb}$ & 79.3 & $1-46(7.2)$ & 5.7 & Lake Wdzydze & $2002-05$ & Rolbiecki 2008 \\
\hline- & $\mathrm{sb}$ & $100^{9}$ & $6-18(12)$ & - & $\begin{array}{l}\text { Lake Raduńskie } \\
\text { Dolne, Lake } \\
\text { Raduńskie } \\
\text { Górne }\end{array}$ & 2004 & $\begin{array}{l}\text { Rolbiecki and } \\
\text { Rokicki } 2005\end{array}$ \\
\hline- & $\mathrm{sb}$ & 58.3 & $2-12(6.0)$ & - & Dead Vistula & 2004 & $\begin{array}{l}\text { Rolbiecki and } \\
\text { Rokicki } 2005\end{array}$ \\
\hline L3, L4, A, e & $\mathrm{sb}$ & 67.8 & $1-37(4.2)$ & & Vistula Lagoon & 2005 & $\begin{array}{l}\text { Rolbiecki and } \\
\text { Rokicki } 2006\end{array}$ \\
\hline L2, L4, A, e & $\mathrm{sb}$ & 65.2 & $1-20(5.5)$ & $3.6^{3}$ & Lake Ostrzyckie & $2005-07$ & Rolbiecki 2011 \\
\hline- & - & - & - & - & Lake Kuc & 2006-07 & $\begin{array}{l}\text { Jeżewski et al. } \\
2007\end{array}$ \\
\hline $\mathrm{L} 2, \mathrm{~A}, \mathrm{e}$ & $\mathrm{sb}$ & 50.0 & $1-12(3.8)$ & $1.9^{3}$ & $\begin{array}{l}\text { Lake } \\
\text { Żarnowieckie }\end{array}$ & 2006-08 & Rolbiecki 2011 \\
\hline L2, A, e & $\mathrm{sb}$ & 40.0 & 2-3 (3.0) & $1.2^{3}$ & $\begin{array}{l}\text { Lake Raduńskie } \\
\text { Dolne, Lake } \\
\text { Raduńskie } \\
\text { Górne }\end{array}$ & 2006-08 & Rolbiecki 2011 \\
\hline $\mathrm{L} 4, \mathrm{~A}$ & $\mathrm{sb}$ & $28.6^{9}$ & $4-6(5.0)$ & $1.4^{3}$ & $\begin{array}{l}\text { Vistula (near } \\
\text { Tczew) }\end{array}$ & 2007 & Rolbiecki 2011 \\
\hline $\mathrm{L} 3, \mathrm{~A}$ & $\begin{array}{l}\text { sb, } \\
\text { intestine } \\
\text { wall }\end{array}$ & $100^{9}$ & $3-6$ & $4.5^{3}$ & $\begin{array}{l}\text { Lake } \\
\text { Żarnowieckie }\end{array}$ & 2007 & Rolbiecki 2011 \\
\hline $\mathrm{pA}, \mathrm{A}$ & $\mathrm{sb}$ & 92.9 & $1-49(10.8)$ & - & Szczecin Lagoon & - & $\begin{array}{l}\text { Popielarczyk et } \\
\text { al. } 2012\end{array}$ \\
\hline $\mathrm{pA}, \mathrm{A}$ & $\mathrm{sb}$ & 64.7 & $1-5(1.9)$ & - & Lake Dąbie & - & $\begin{array}{l}\text { Popielarczyk et } \\
\text { al. } 2012\end{array}$ \\
\hline $\mathrm{pA}, \mathrm{A}$ & $\mathrm{sb}$ & 64.2 & $3-62(18.5)$ & - & Lake Bukowo & - & $\begin{array}{l}\text { Popielarczyk et } \\
\text { al. } 2012\end{array}$ \\
\hline $\mathrm{pA}, \mathrm{A}$ & $\mathrm{sb}$ & $100^{9}$ & $1-50(13.6)$ & - & Lake Łebsko & - & $\begin{array}{l}\text { Popielarczyk et } \\
\text { al. } 2012\end{array}$ \\
\hline $\mathrm{pA}, \mathrm{A}$ & $\mathrm{sb}$ & $80.0^{9}$ & 3-16 (8.0) & - & Lake Gardno & - & $\begin{array}{l}\text { Popielarczyk et } \\
\text { al. } 2012\end{array}$ \\
\hline $\mathrm{pA}, \mathrm{A}$ & $\mathrm{sb}$ & 85.7 & $1-23(7.2)$ & - & Lake Resko & - & $\begin{array}{l}\text { Popielarczyk et } \\
\text { al. } 2012\end{array}$ \\
\hline $\mathrm{pA}, \mathrm{A}$ & $\mathrm{sb}$ & 76.9 & $1-55(11.6)$ & - & Lake Jamno & - & $\begin{array}{l}\text { Popielarczyk et } \\
\text { al. } 2012\end{array}$ \\
\hline $\mathrm{pA}$ & $\mathrm{sb}$ & 50.0 & $1-7(3.2)$ & - & Oder & - & $\begin{array}{l}\text { Popielarczyk et } \\
\text { al. } 2012\end{array}$ \\
\hline $\mathrm{pA}, \mathrm{A}$ & $\mathrm{sb}$ & 71.4 & $1-6(3.4)$ & - & Vistula & - & $\begin{array}{l}\text { Popielarczyk et } \\
\text { al. } 2012\end{array}$ \\
\hline
\end{tabular}




\begin{tabular}{|c|c|c|c|c|c|c|c|}
\hline $\begin{array}{l}\text { Parasite } \\
\text { life } \\
\text { stage }\end{array}$ & $\begin{array}{l}\text { Micro } \\
\text { habitat }\end{array}$ & $\begin{array}{l}\mathbf{P} \\
{[\%]}\end{array}$ & $\begin{array}{l}\text { Intensity } \\
\text { range } \\
\text { (mean) }\end{array}$ & $\begin{array}{l}\text { Abun } \\
\text { dance }\end{array}$ & Locality & $\begin{array}{l}\text { Material } \\
\text { collection } \\
\text { year }\end{array}$ & References \\
\hline $\mathrm{pA}$ & $\mathrm{sb}$ & 62.5 & $1-4(1.7)$ & - & River Węgorapa & - & $\begin{array}{l}\text { Popielarczyk et } \\
\text { al. } 2012\end{array}$ \\
\hline $\mathrm{pA}, \mathrm{A}$ & $\mathrm{sb}$ & 66.7 & $1-7(2.6)$ & - & River Drwęca & - & $\begin{array}{l}\text { Popielarczyk et } \\
\text { al. } 2012\end{array}$ \\
\hline A & $\mathrm{sb}$ & $100^{9}$ & $7(7.0)$ & 7.0 & River Piaśnica & $2014-16$ & This study \\
\hline A, L4 & $\mathrm{sb}$ & $40.0^{9}$ & $6-9(7.5)$ & 3.0 & River Szkarpawa & 2014-16 & This study \\
\hline A & $\mathrm{sb}$ & $42.9^{9}$ & $2-4(3.0)$ & 1.28 & Lake Sarbsko & 2016 & This study \\
\hline \multicolumn{8}{|c|}{ Family Camallanidae } \\
\hline \multicolumn{8}{|c|}{ Camallanus lacustris (Zoega, 1776) } \\
\hline- & intestine & $20.0^{3,9}$ & $1(1.0)$ & $0.20^{3}$ & $\begin{array}{l}\text { Vistula (near } \\
\text { Warszawa) }\end{array}$ & $1924-25$ & Dąbrowska 1970 \\
\hline- & intestine & $100^{3,9}$ & $87\left(87.0^{3}\right)$ & $87.0^{3}$ & Lake Wdzydze & 1958 & $\begin{array}{l}\text { Grabda et al. } \\
1961\end{array}$ \\
\hline- & intestine & - & - & - & $\begin{array}{l}\text { Lake Gardno, } \\
\text { River Nogat } \\
\text { (near Tczew), } \\
\text { Lake Kalwa }\end{array}$ & - & Grabda 1971 \\
\hline- & - & 15 & $1-4\left(2.7^{3}\right)$ & $0.40^{3}$ & $\begin{array}{l}\text { Oder (near } \\
\text { Stołczyn) }\end{array}$ & 1971 & Seyda 1973 \\
\hline- & - & 3 & $12(12)$ & $0.39^{3}$ & Lake Dąbie & 1971 & Seyda 1973 \\
\hline- & - & 45.5 & $\left(4.2^{3}\right)$ & 1.9 & Lake Dgał Wielki & $1979-84$ & $\begin{array}{l}\text { Grabda- } \\
\text { Kazubska et al. } \\
1987\end{array}$ \\
\hline A & intestine & $3.1^{3}$ & $1-7\left(3.4^{3}\right)$ & $0.11^{3}$ & Szczecin Lagoon & $1982-83$ & $\begin{array}{l}\text { Orecka-Grabda } \\
\text { and Wierzbicka } \\
1994\end{array}$ \\
\hline $\mathrm{L} 4, \mathrm{~A}$ & intestine & 5.00 & $1-3$ & 0.057 & Dead Vistula & $1982-90$ & $\begin{array}{l}\text { Sulgostowska } \\
1993\end{array}$ \\
\hline $\mathrm{L} 4, \mathrm{~A}$ & intestine & 2.93 & $1-20$ & 0.152 & Gulf of Gdańsk & $1982-90$ & $\begin{array}{l}\text { Sulgostowska } \\
1993\end{array}$ \\
\hline- & - & 7 & - & - & $\begin{array}{l}\text { Lake Dabrowa } \\
\text { Wielka }\end{array}$ & $1990-91$ & $\begin{array}{l}\text { Własow et al. } \\
1991\end{array}$ \\
\hline- & intestine & - & - & - & Lake lńsko & 1993 & $\begin{array}{l}\text { Rząd and } \\
\text { Pilecka-Rapacz } \\
2002\end{array}$ \\
\hline- & intestine & 10 & $1(1)$ & - & Lake Miedwie & $1997-99$ & $\begin{array}{l}\text { Sobecka and } \\
\text { Piasecki } 2002\end{array}$ \\
\hline- & intestine & 3.41 & $1-2(1.67)$ & 0.06 & Lake Łebsko & 2000-06 & $\begin{array}{l}\text { Morozińska- } \\
\text { Gogol } 2011\end{array}$ \\
\hline- & - & 3.8 & $1-7(2.0)$ & - & Puck Bay & 2002 & $\begin{array}{l}\text { Bystydzieńska et } \\
\text { al. } 2005\end{array}$ \\
\hline
\end{tabular}




\begin{tabular}{|c|c|c|c|c|c|c|c|}
\hline $\begin{array}{l}\text { Parasite } \\
\text { life } \\
\text { stage }\end{array}$ & $\begin{array}{l}\text { Micro } \\
\text { habitat }\end{array}$ & $\begin{array}{l}\mathbf{P} \\
{[\%]}\end{array}$ & $\begin{array}{l}\text { Intensity } \\
\text { range } \\
\text { (mean) }\end{array}$ & $\begin{array}{l}\text { Abun } \\
\text { dance }\end{array}$ & Locality & $\begin{array}{l}\text { Material } \\
\text { collection } \\
\text { year }\end{array}$ & References \\
\hline A & stomach & 2.2 & 2 & 0.04 & Lake Wdzydze & 2004 & This study \\
\hline A & - & 2.2 & $2(2)$ & $0.04^{3}$ & Vistula Lagoon & 2005 & $\begin{array}{l}\text { Rolbiecki and } \\
\text { Rokicki } 2006\end{array}$ \\
\hline A & intestine & $25.0^{9}$ & $7(7.0)$ & 1.75 & Lake Kłodno & 2015 & This study \\
\hline \multicolumn{8}{|c|}{ Camallanus truncatus (Rudolphi, 1814) } \\
\hline- & - & 3 & $1(1)$ & 0.033 & Szczecin Lagoon & 1971 & Seyda 1973 \\
\hline L4 & - & 0.29 & $1-3$ & 0.005 & Gulf of Gdańsk & $1982-90$ & $\begin{array}{l}\text { Sulgostowska } \\
1993\end{array}$ \\
\hline A & - & 2.2 & $2-4(3)$ & 0.073 & Vistula Lagoon & 2005 & $\begin{array}{l}\text { Rolbiecki and } \\
\text { Rokicki } 2006\end{array}$ \\
\hline A & intestine & $10.0^{9}$ & $4(4.0)$ & 0.40 & $\begin{array}{l}\text { Lake } \\
\text { Choczewskie }\end{array}$ & $2008-15$ & This study \\
\hline A & intestine & $25.0^{9}$ & $1(1.0)$ & 0.25 & Lake Kłodno & 2015 & This study \\
\hline A & intestine & $20.0^{9}$ & $1(1.0)$ & 0.20 & Lake Dargin & 2015 & This study \\
\hline \multicolumn{8}{|c|}{ Family Cystidicolidae } \\
\hline \multicolumn{8}{|c|}{ Cystidicola farionis Fischer, 1798} \\
\hline- & - & 0.7 & $7(7)$ & $0.05^{3}$ & Puck Bay & 2002 & $\begin{array}{l}\text { Bystydzieńska et } \\
\text { al. } 2005\end{array}$ \\
\hline \multicolumn{8}{|c|}{ Spinitectus inermis (Zeder, 1800) } \\
\hline $\mathrm{F}$ & intestine & $3.7^{3}$ & $3(3)$ & $0.11^{3}$ & $\begin{array}{l}\text { Baltic Sea (near } \\
\text { Chłapowo), Puck } \\
\text { Bay }\end{array}$ & 1930-31 & Markowski 1933 \\
\hline$A, L$ & intestine & 3.3 & $1-5\left(3.0^{3}\right)$ & $0.10^{3}$ & $\begin{array}{l}\text { River Wieprza } \\
\text { (near Darłowo) }\end{array}$ & $\begin{array}{l}1999 \\
2001\end{array}$ & $\begin{array}{l}\text { Pilecka-Rapacz } \\
\text { and Sobecka } \\
2004\end{array}$ \\
\hline$A, L$ & intestine & 6.1 & $1-10\left(3.4^{3}\right)$ & $0.21^{3}$ & $\begin{array}{l}\text { River Rega } \\
\text { (Lake Rejowice) }\end{array}$ & $1999-2003$ & $\begin{array}{l}\text { Pilecka-Rapacz } \\
\text { and Sobecka } \\
2004\end{array}$ \\
\hline$A, L$ & intestine & 10.9 & $1-6\left(2.3^{3}\right)$ & $0.25^{3}$ & River Radew & $2000-01$ & $\begin{array}{l}\text { Pilecka-Rapacz } \\
\text { and Sobecka } \\
2004\end{array}$ \\
\hline \multicolumn{8}{|c|}{ Family Daniconematidae } \\
\hline \multicolumn{8}{|c|}{ Daniconema anguillae Moravec \& Køie, 1987} \\
\hline- & gills & $1.9^{3}$ & $1(1.0)$ & $0.02^{3}$ & $\begin{array}{l}\text { Lake Dabrowa } \\
\text { Wielka }\end{array}$ & $1990-91$ & $\begin{array}{l}\text { Własow et al. } \\
1991\end{array}$ \\
\hline $\mathrm{F}$ & sb wall & - & 1 & - & $\begin{array}{l}\text { Reservoir near } \\
\text { village Gaj }\end{array}$ & 1993 & $\begin{array}{l}\text { Własow et al. } \\
1991\end{array}$ \\
\hline \multicolumn{8}{|c|}{ Family Dioctophymatidae } \\
\hline
\end{tabular}




\begin{tabular}{|c|c|c|c|c|c|c|c|}
\hline $\begin{array}{l}\text { Parasite } \\
\text { life } \\
\text { stage }\end{array}$ & $\begin{array}{l}\text { Micro } \\
\text { habitat }\end{array}$ & $\begin{array}{l}\mathbf{P} \\
{[\%]}\end{array}$ & $\begin{array}{l}\text { Intensity } \\
\text { range } \\
\text { (mean) }\end{array}$ & $\begin{array}{l}\text { Abun } \\
\text { dance }\end{array}$ & Locality & $\begin{array}{l}\text { Material } \\
\text { collection } \\
\text { year }\end{array}$ & References \\
\hline L & $\begin{array}{l}\text { intestine } \\
\text { wall }\end{array}$ & 3 & 3 & $0.09^{3}$ & Szczecin Lagoon & 1971 & Seyda 1973 \\
\hline L & $\begin{array}{l}\text { stomach, } \\
\text { body cavity }\end{array}$ & $2.5^{3}$ & $1-3\left(2.2^{3}\right)$ & $0.06^{3}$ & Szczecin Lagoon & $1982-83$ & $\begin{array}{l}\text { Orecka-Grabda } \\
\text { and Wierzbicka } \\
1994\end{array}$ \\
\hline \multicolumn{8}{|c|}{ Family Raphidascarididae } \\
\hline \multicolumn{8}{|c|}{ Hysterothylacium aduncum (Rudolphi, 1802) } \\
\hline- & intestine & $33.3^{3,9}$ & $1(1.0)$ & $0.33^{3}$ & River Gnilna & - & Grabda 1971 \\
\hline- & - & 0.2 & $1(1)$ & $0.002^{3}$ & Vistula Lagoon & $2001-02$ & $\begin{array}{l}\text { Bystydzieńska et } \\
\text { al. } 2005\end{array}$ \\
\hline- & - & 0.7 & $1(1)$ & $0.01^{3}$ & Puck Bay & 2002 & $\begin{array}{l}\text { Bystydzieńska et } \\
\text { al. } 2005\end{array}$ \\
\hline A & - & 2.2 & $1-2(1.5)$ & $0.03^{3}$ & Vistula Lagoon & 2005 & $\begin{array}{l}\text { Rolbiecki and } \\
\text { Rokicki } 2006\end{array}$ \\
\hline \multicolumn{8}{|c|}{ Raphidascaris acus (Bloch, 1779) } \\
\hline- & intestine & $20.0^{3,9}$ & $1(1)$ & $0.20^{3}$ & $\begin{array}{l}\text { Vistula (near } \\
\text { Warszawa) }\end{array}$ & $1924-25$ & Dąbrowska 1970 \\
\hline M & intestine & $3.7^{3}$ & $1(1)$ & $0.04^{3}$ & $\begin{array}{l}\text { Baltic Sea (near } \\
\text { Chłapowo), Puck } \\
\text { Bay }\end{array}$ & 1930-31 & Markowski 1933 \\
\hline- & intestine & $100^{3,9}$ & $1(1.0)$ & $1.00^{3}$ & Lake Druzno & 1951 & Kozicka 1959 \\
\hline- & intestine & - & - & - & $\begin{array}{l}\text { River Gnilna, } \\
\text { Lake Blanki }\end{array}$ & - & Grabda 1971 \\
\hline- & - & 10 & $1-2\left(1.5^{3}\right)$ & $0.15^{3}$ & $\begin{array}{l}\text { Oder (near } \\
\text { Stołczyn) }\end{array}$ & 1971 & Seyda 1973 \\
\hline- & - & 6 & $3-4\left(3.5^{3}\right)$ & $0.22^{3}$ & Szczecin Lagoon & 1971 & Seyda 1973 \\
\hline - & - & 13 & $1-6\left(2.5^{3}\right)$ & $0.32^{3}$ & Lake Dąbie & 1971 & Seyda 1973 \\
\hline A & - & 9.1 & $1^{3}\left(1^{3}\right)$ & 0.09 & Lake Dgał Wielki & $1979-84$ & $\begin{array}{l}\text { Grabda- } \\
\text { Kazubska et al. } \\
1987\end{array}$ \\
\hline A & intestine & $2.5^{3}$ & $1-6\left(4.0^{3}\right)$ & $0.10^{3}$ & Szczecin Lagoon & $1982-83$ & $\begin{array}{l}\text { Orecka-Grabda } \\
\text { and Wierzbicka } \\
1994\end{array}$ \\
\hline - & - & 4.2 & $2(2)$ & 0.08 & Skolwiński Canal & $1982-83$ & $\begin{array}{l}\text { Orecka-Grabda } \\
\text { and Wierzbicka } \\
1994\end{array}$ \\
\hline $\mathrm{L} 4, \mathrm{~A}$ & intestine & 24.28 & $1-26$ & 0.900 & Dead Vistula & $1982-90$ & $\begin{array}{l}\text { Sulgostowska } \\
1993\end{array}$ \\
\hline $\mathrm{L} 4, \mathrm{~A}$ & intestine & 19.06 & $1-68$ & 1.067 & Gulf of Gdańsk & $1982-90$ & $\begin{array}{l}\text { Sulgostowska } \\
1993\end{array}$ \\
\hline $\mathrm{L} 4, \mathrm{~A}$ & intestine & 9.09 & $1-2$ & 0.107 & $\begin{array}{l}\text { Baltic Sea (near } \\
\text { Władysławowo) }\end{array}$ & $1982-90$ & $\begin{array}{l}\text { Sulgostowska } \\
1993\end{array}$ \\
\hline
\end{tabular}




\begin{tabular}{|c|c|c|c|c|c|c|c|}
\hline $\begin{array}{l}\text { Parasite } \\
\text { life } \\
\text { stage }\end{array}$ & $\begin{array}{l}\text { Micro } \\
\text { habitat }\end{array}$ & $\begin{array}{l}\mathbf{P} \\
{[\%]}\end{array}$ & $\begin{array}{l}\text { Intensity } \\
\text { range } \\
\text { (mean) }\end{array}$ & $\begin{array}{l}\text { Abun } \\
\text { dance }\end{array}$ & Locality & $\begin{array}{l}\text { Material } \\
\text { collection } \\
\text { year }\end{array}$ & References \\
\hline- & intestine & - & - & - & Lake Ińsko & 1993 & $\begin{array}{l}\text { Rząd and } \\
\text { Pilecka-Rapacz } \\
2002\end{array}$ \\
\hline L & intestine & $0.4^{3}$ & $1(1.0)$ & $0.003^{3}$ & $\begin{array}{l}\text { Rivers Rega } \\
\text { (Lake Rejowice), } \\
\text { Radew, Wieprza } \\
\text { (near Darłowo) }\end{array}$ & $1999-2003$ & $\begin{array}{l}\text { Pilecka-Rapacz } \\
\text { and Sobecka } \\
2004\end{array}$ \\
\hline- & intestine & 1.14 & $1(1.00)$ & 0.01 & Lake Łebsko & $2000-06$ & $\begin{array}{l}\text { Morozińska- } \\
\text { Gogol } 2011\end{array}$ \\
\hline- & - & 56.1 & $1-92(5.6)$ & - & Vistula Lagoon & 2001-02 & $\begin{array}{l}\text { Bystydzieńska et } \\
\text { al. } 2005\end{array}$ \\
\hline- & - & 4.5 & $1-12(5)$ & - & Puck Bay & 2002 & $\begin{array}{l}\text { Bystydzieńska et } \\
\text { al. } 2005\end{array}$ \\
\hline A & intestine & 2.2 & $2(1.0)$ & 0.02 & Lake Wdzydze & 2004 & This study \\
\hline \multicolumn{8}{|c|}{ Family Quimperiidae } \\
\hline \multicolumn{8}{|c|}{ Paraquimperia tenerrima (von Linstow, 1878) } \\
\hline L & intestine & 0.4 & 1 & 0.0033 & $\begin{array}{l}\text { Rivers Rega } \\
\text { (Lake Rejowice), } \\
\text { Radew, Wieprza } \\
\text { (near Darłowo) }\end{array}$ & $1999-2003$ & $\begin{array}{l}\text { Pilecka-Rapacz } \\
\text { and Sobecka } \\
2004\end{array}$ \\
\hline \multicolumn{8}{|c|}{ ACANTHOCEPHALA } \\
\hline \multicolumn{8}{|c|}{ Family Echinorhynchidae } \\
\hline \multicolumn{8}{|c|}{ Acanthocephalus anguillae (Müller, 1780) } \\
\hline- & intestine & $20.0^{3,9}$ & $1(1)$ & $0.20^{3}$ & $\begin{array}{l}\text { Vistula (near } \\
\text { Warszawa) }\end{array}$ & $1924-25$ & Dąbrowska 1970 \\
\hline- & intestine & $55.5^{3,9}$ & $1-7$ & - & Potok Oliwski & - & Grabda 1971 \\
\hline- & intestine & $33.3^{3,9}$ & 8 & $2.67^{3}$ & River Łupawa & - & Grabda 1971 \\
\hline- & intestine & $36.4^{3}$ & $1-9$ & - & $\begin{array}{l}\text { Vistula (near } \\
\text { Tczew) }\end{array}$ & - & Grabda 1971 \\
\hline- & intestine & $40.0^{3,9}$ & 1 & $0.40^{3}$ & $\begin{array}{l}\text { River Nogat } \\
\text { (near Malbork) }\end{array}$ & - & Grabda 1971 \\
\hline- & intestine & - & - & - & Lake Probark & - & Grabda 1971 \\
\hline- & - & 35 & $1-37\left(9.1^{3}\right)$ & $3.20^{3}$ & $\begin{array}{l}\text { Oder (near } \\
\text { Stołczyn) }\end{array}$ & 1971 & Seyda 1973 \\
\hline- & - & 29 & $1-74\left(11.1^{3}\right)$ & $3.23^{3}$ & Lake Dąbie & 1971 & Seyda 1973 \\
\hline- & intestine & $2.5^{3}$ & $1-2\left(1.2^{3}\right)$ & $0.03^{3}$ & Szczecin Lagoon & $1982-83$ & $\begin{array}{l}\text { Orecka-Grabda } \\
\text { and Wierzbicka } \\
1994\end{array}$ \\
\hline- & intestine & 4.2 & $17(17)$ & 0.71 & Skolwiński Canal & $1982-83$ & $\begin{array}{l}\text { Orecka-Grabda } \\
\text { and Wierzbicka } \\
1994\end{array}$ \\
\hline
\end{tabular}




\begin{tabular}{|c|c|c|c|c|c|c|c|}
\hline $\begin{array}{l}\text { Parasite } \\
\text { life } \\
\text { stage }\end{array}$ & $\begin{array}{l}\text { Micro } \\
\text { habitat }\end{array}$ & $\begin{array}{l}\mathbf{P} \\
{[\%]}\end{array}$ & $\begin{array}{l}\text { Intensity } \\
\text { range } \\
\text { (mean) }\end{array}$ & $\begin{array}{l}\text { Abun } \\
\text { dance }\end{array}$ & Locality & $\begin{array}{l}\text { Material } \\
\text { collection } \\
\text { year }\end{array}$ & References \\
\hline A & intestine & 4.29 & $1-9$ & 0.157 & Dead Vistula & $1982-90$ & $\begin{array}{l}\text { Sulgostowska } \\
1993\end{array}$ \\
\hline A & intestine & 1.61 & $1-23$ & 0.061 & Gulf of Gdańsk & $1982-90$ & $\begin{array}{l}\text { Sulgostowska } \\
1993\end{array}$ \\
\hline- & intestine & 43.4 & - & - & Lake Ińsko & 1993 & $\begin{array}{l}\text { Rząd and } \\
\text { Pilecka-Rapacz } \\
2002\end{array}$ \\
\hline - & intestine & 7.95 & $1-21(6.57)$ & 0.52 & Lake Łebsko & $2000-06$ & $\begin{array}{l}\text { Morozińska- } \\
\text { Gogol } 2011\end{array}$ \\
\hline - & - & 0.6 & $1-8(3.3)$ & - & Vistula Lagoon & 2001-02 & $\begin{array}{l}\text { Bystydzieńska et } \\
\text { al. } 2005\end{array}$ \\
\hline- & - & 1.5 & $1(1)$ & $0.01^{3}$ & Puck Bay & 2002 & $\begin{array}{l}\text { Bystydzieńska et } \\
\text { al. } 2005\end{array}$ \\
\hline \multicolumn{8}{|c|}{ Acanthocephalus lucii (Müller, 1776) } \\
\hline- & intestine & $100^{3,9}$ & $2(2.0)$ & $2.00^{3}$ & Lake Druzno & 1951 & Styczyńska 1958 \\
\hline- & intestine & $100^{3,9}$ & $2(2.0)$ & $2.00^{3}$ & Lake Druzno & 1951 & Kozicka 1959 \\
\hline- & intestine & $100^{3,9}$ & $7\left(7.0^{3}\right)$ & $7.00^{3}$ & Lake Wdzydze & 1958 & $\begin{array}{l}\text { Grabda et al. } \\
1961\end{array}$ \\
\hline- & intestine & - & - & - & $\begin{array}{l}\text { Vistula mouth, } \\
\text { River Łupawa, } \\
\text { River Dadaj, } \\
\text { Lake Dąbrowa } \\
\text { Wielka }\end{array}$ & - & Grabda 1971 \\
\hline- & - & 15 & $2-8\left(4.0^{3}\right)$ & $0.60^{3}$ & $\begin{array}{l}\text { Oder (near } \\
\text { Stołczyn) }\end{array}$ & 1971 & Seyda 1973 \\
\hline- & - & 3 & $1(1)$ & $0.03^{3}$ & Szczecin Lagoon & 1971 & Seyda 1973 \\
\hline- & - & 10 & $1(1)$ & $0.10^{3}$ & Lake Dąbie & 1971 & Seyda 1973 \\
\hline- & intestine & $5.0-16.7$ & $1-7\left(2.4^{3}\right)$ & $0.25^{3}$ & Szczecin Lagoon & $1982-83$ & $\begin{array}{l}\text { Orecka-Grabda } \\
\text { and Wierzbicka } \\
1994\end{array}$ \\
\hline- & intestine & 16.7 & $1-11\left(3.8^{3}\right)$ & 0.62 & Skolwiński Canal & $1982-83$ & $\begin{array}{l}\text { Orecka-Grabda } \\
\text { and Wierzbicka } \\
1994\end{array}$ \\
\hline A & - & 3.57 & $1-5$ & 0.092 & Dead Vistula & $1982-90$ & $\begin{array}{l}\text { Sulgostowska } \\
1993\end{array}$ \\
\hline$A$ & - & 0.88 & $1-7$ & 0.024 & Gulf of Gdańsk & $1982-90$ & $\begin{array}{l}\text { Sulgostowska } \\
1993\end{array}$ \\
\hline- & - & 6 & - & - & Lake Śniardwy & 1989 & $\begin{array}{l}\text { Własow et al. } \\
1991\end{array}$ \\
\hline- & - & 6 & - & - & Lake Mamry & 1990 & $\begin{array}{l}\text { Własow et al. } \\
1991\end{array}$ \\
\hline- & - & 6 & - & - & $\begin{array}{l}\text { Lake Dabrowa } \\
\text { Wielka }\end{array}$ & $1990-91$ & $\begin{array}{l}\text { Własow et al. } \\
1991\end{array}$ \\
\hline
\end{tabular}




\begin{tabular}{|c|c|c|c|c|c|c|c|}
\hline $\begin{array}{l}\text { Parasite } \\
\text { life } \\
\text { stage }\end{array}$ & $\begin{array}{l}\text { Micro } \\
\text { habitat }\end{array}$ & $\begin{array}{l}\mathbf{P} \\
{[\%]}\end{array}$ & $\begin{array}{l}\text { Intensity } \\
\text { range } \\
\text { (mean) }\end{array}$ & $\begin{array}{l}\text { Abun } \\
\text { dance }\end{array}$ & Locality & $\begin{array}{l}\text { Material } \\
\text { collection } \\
\text { year }\end{array}$ & References \\
\hline- & - & 0.2 & $14(14)$ & $0.03^{3}$ & Vistula Lagoon & $2001-02$ & $\begin{array}{l}\text { Bystydzieńska et } \\
\text { al. } 2005\end{array}$ \\
\hline- & intestine & 15.91 & $1-80(14.14)$ & 2.41 & Lake Łebsko & $2000-06$ & $\begin{array}{l}\text { Morozińska- } \\
\text { Gogol } 2011\end{array}$ \\
\hline- & - & 0.7 & $14(14)$ & $0.11^{3}$ & Puck Bay & 2002 & $\begin{array}{l}\text { Bystydzieńska et } \\
\text { al. } 2005\end{array}$ \\
\hline A & intestine & 28.3 & $1-8(2.6)$ & 0.74 & Lake Wdzydze & 2004 & This study \\
\hline A & - & 1.1 & 1 & $0.01^{3}$ & Vistula Lagoon & 2005 & $\begin{array}{l}\text { Rolbiecki and } \\
\text { Rokicki } 2006\end{array}$ \\
\hline \multicolumn{8}{|c|}{ Echinorhynchus gadi Zoega in Müller, 1776} \\
\hline A & - & 0.73 & $1-4$ & 0.014 & Gulf of Gdańsk & $1982-90$ & $\begin{array}{l}\text { Sulgostowska } \\
1993\end{array}$ \\
\hline- & - & 0.2 & $1(1)$ & $0.002^{3}$ & Vistula Lagoon & 2001-02 & $\begin{array}{l}\text { Bystydzieńska et } \\
\text { al. } 2005\end{array}$ \\
\hline- & - & 3 & $1-10(3.2)$ & - & Puck Bay & 2002 & $\begin{array}{l}\text { Bystydzieńska et } \\
\text { al. } 2005\end{array}$ \\
\hline \multicolumn{8}{|c|}{ Echinorhynchus truttae (Schrank, 1788) } \\
\hline- & intestine & $33.3^{3,9}$ & $3(3.0)$ & $1.00^{3}$ & River Łupawa & - & Grabda 1971 \\
\hline- & intestine & $11.1^{3,9}$ & $1(1.0)$ & $0.11^{3}$ & Potok Oliwski & - & Grabda 1971 \\
\hline- & intestine & 3.41 & $2-21(8.67)$ & 0.30 & Lake Łebsko & $2000-06$ & $\begin{array}{l}\text { Morozińska- } \\
\text { Gogol } 2011\end{array}$ \\
\hline \multicolumn{8}{|c|}{ Family Neoechinorhynchidae } \\
\hline \multicolumn{8}{|c|}{ Neoechinorhynchus rutili (Müller, 1780) } \\
\hline- & intestine & $5.9^{3}$ & 2 & $0.12^{3}$ & Gulf of Gdańsk & $1967-71$ & Rokicki 1975 \\
\hline- & intestine & 2.27 & $1-2(1.5)$ & 0.03 & Lake Łebsko & $2000-06$ & $\begin{array}{l}\text { Morozińska- } \\
\text { Gogol } 2011\end{array}$ \\
\hline \multicolumn{8}{|c|}{ Family Polymorphidae } \\
\hline \multicolumn{8}{|c|}{ Corynosoma strumosum (Rudolphi, 1802) } \\
\hline cystacanth & - & 1.1 & $1(1)$ & $0.01^{3}$ & Vistula Lagoon & 2005 & $\begin{array}{l}\text { Rolbiecki and } \\
\text { Rokicki } 2006\end{array}$ \\
\hline \multicolumn{8}{|c|}{ Family Pomphorhynchidae } \\
\hline \multicolumn{8}{|c|}{ Pomphorhynchus laevis (Zoega in Müller, 1776) } \\
\hline A & intestine & $14.8^{3}$ & $1-37\left(10.8^{3}\right)$ & $1.59^{3}$ & $\begin{array}{l}\text { Baltic Sea (near } \\
\text { Chłapowo), Puck } \\
\text { Bay }\end{array}$ & $1930-31$ & Markowski 1933 \\
\hline- & - & 3 & $1(1)$ & $0.03^{3}$ & Lake Dąbie & 1971 & Seyda 1973 \\
\hline A & intestine & 0.44 & $1-2$ & 0.005 & Gulf of Gdańsk & $1982-90$ & $\begin{array}{l}\text { Sulgostowska } \\
1993\end{array}$ \\
\hline A & intestine & 1.65 & $1-3$ & 0.033 & Baltic Sea & $1982-90$ & $\begin{array}{l}\text { Sulgostowska } \\
1993\end{array}$ \\
\hline
\end{tabular}




\begin{tabular}{|c|c|c|c|c|c|c|c|}
\hline $\begin{array}{l}\text { Parasite } \\
\text { life } \\
\text { stage }\end{array}$ & $\begin{array}{l}\text { Micro } \\
\text { habitat }\end{array}$ & $\begin{array}{l}\mathbf{P} \\
{[\%]}\end{array}$ & $\begin{array}{l}\text { Intensity } \\
\text { range } \\
\text { (mean) }\end{array}$ & $\begin{array}{l}\text { Abun } \\
\text { dance }\end{array}$ & Locality & $\begin{array}{l}\text { Material } \\
\text { collection } \\
\text { year }\end{array}$ & References \\
\hline- & - & 6 & $1-10(2.8)$ & - & Puck Bay & 2002 & $\begin{array}{l}\text { Bystydzieńska et } \\
\text { al. } 2005\end{array}$ \\
\hline- & intestine & 3.41 & $1-2(1.3)$ & 0.05 & Lake Łebsko & $2000-06$ & $\begin{array}{l}\text { Morozińska- } \\
\text { Gogol } 2011\end{array}$ \\
\hline \multicolumn{8}{|c|}{ Family Tenuisentidae } \\
\hline \multicolumn{8}{|c|}{ Paratenuisentis ambiguus Van Cleave, 1923} \\
\hline A & intestine & 6.82 & $1-163(28.17)$ & 1.92 & Lake Łebsko & $2000-06$ & $\begin{array}{l}\text { Morozińska- } \\
\text { Gogol (2008), } \\
\text { Morozińska- } \\
\text { Gogol (2011), } \\
\text { Morozińska- } \\
\text { Gogol (2009) }\end{array}$ \\
\hline \multicolumn{8}{|c|}{ MOLLUSCA: BIVALVIA } \\
\hline \multicolumn{8}{|c|}{ Family Unionidae } \\
\hline glochidium & - & 9.1 & $1^{3}\left(1^{3}\right)$ & 0.09 & Lake Dgał Wielki & 1979-84 & $\begin{array}{l}\text { Grabda- } \\
\text { Kazubska et al. } \\
1987\end{array}$ \\
\hline glochidium & - & 1.14 & $21(21)$ & 0.24 & Lake Łebsko & $2000-06$ & $\begin{array}{l}\text { Morozińska- } \\
\text { Gogol } 2011\end{array}$ \\
\hline glochidium & gills & $33.3^{9}$ & $6(6.0)$ & 2.0 & River Słupia & 2015-16 & This study \\
\hline \multicolumn{8}{|c|}{ ANNELIDA: CLITELLATA } \\
\hline \multicolumn{8}{|c|}{ Family Piscicolidae } \\
\hline \multicolumn{8}{|c|}{ Piscicola geometra (Linnaeus, 1761) } \\
\hline- & - & 1.1 & 1 & $0.01^{3}$ & Vistula Lagoon & 2005 & $\begin{array}{l}\text { Rolbiecki and } \\
\text { Rokicki } 2006\end{array}$ \\
\hline \multicolumn{8}{|c|}{ Piscicola respirans Troschel, 1850} \\
\hline- & - & - & - & - & $\begin{array}{l}\text { River Dunajec } \\
\text { with tributaries }\end{array}$ & - & Sitowski 1937 \\
\hline \multicolumn{8}{|c|}{ ARTHROPODA: BRANCHIURA } \\
\hline \multicolumn{8}{|c|}{ Family Argulidae } \\
\hline \multicolumn{8}{|c|}{ Argulus foliaceus (Linnaeus, 1758) } \\
\hline- & gills & $1.9^{3}$ & $1(1)$ & $0.02^{3}$ & Szczecin Lagoon & $1982-83$ & $\begin{array}{l}\text { Orecka-Grabda } \\
\text { and Wierzbicka } \\
1994\end{array}$ \\
\hline \multicolumn{8}{|c|}{ ARTHROPODA: COPEPODA } \\
\hline \multicolumn{8}{|c|}{ Family Ergasilidae } \\
\hline \multicolumn{8}{|c|}{ Ergasilus gibbus Nordmann, 1832} \\
\hline- & gills & - & - & - & Vistula Lagoon & - & Zaddach 1844 \\
\hline A & gills & 28.5 & - & - & Vistula Lagoon & 1908 & Wegener 1909 \\
\hline
\end{tabular}




\begin{tabular}{|c|c|c|c|c|c|c|c|}
\hline $\begin{array}{l}\text { Parasite } \\
\text { life } \\
\text { stage }\end{array}$ & $\begin{array}{l}\text { Micro } \\
\text { habitat }\end{array}$ & $\begin{array}{l}\mathbf{P} \\
{[\%]}\end{array}$ & $\begin{array}{l}\text { Intensity } \\
\text { range } \\
\text { (mean) }\end{array}$ & $\begin{array}{l}\text { Abun } \\
\text { dance }\end{array}$ & Locality & $\begin{array}{l}\text { Material } \\
\text { collection } \\
\text { year }\end{array}$ & References \\
\hline- & - & 16.6 & $(1.4)$ & $0.23^{3}$ & Lake Dąbie & 1955 & $\begin{array}{l}\text { Kozikowska } \\
1957\end{array}$ \\
\hline- & gills & 38.3 & $1-51(8.5)$ & $3.21^{3}$ & Vistula Lagoon & 1959 & Grabda 1962 \\
\hline- & - & - & - & - & Puck Bay & 1959 & Grabda 1962 \\
\hline- & gills & 27.6 & up to $4(2.3)$ & - & Puck Bay & 1959 & $\begin{array}{l}\text { Kozikowska } \\
1965\end{array}$ \\
\hline- & gills & $75.0^{3,9}$ & up to 75 & - & $\begin{array}{l}\text { Vistula (near } \\
\text { Świbno) }\end{array}$ & $1961-63$ & Grabda 1972 \\
\hline- & - & - & - & - & Vistula mouth & - & Grabda 1967 \\
\hline- & gills & $1.9^{3}$ & $1-4\left(3.0^{3}\right)$ & $0.06^{3}$ & Szczecin Lagoon & $1982-83$ & $\begin{array}{l}\text { Orecka-Grabda } \\
\text { and Wierzbicka } \\
1994\end{array}$ \\
\hline \multicolumn{8}{|c|}{ Ergasilus sieboldi von Nordmann, 1832} \\
\hline- & gills & 38.3 & $1-12(3.2)$ & $1.25^{3}$ & Vistula Lagoon & 1959 & Grabda 1962 \\
\hline- & - & - & - & - & $\begin{array}{l}\text { Lake Dąbrowa } \\
\text { Wielka }\end{array}$ & - & Grabda 1962 \\
\hline- & - & 45.5 & $\left(4.4^{3}\right)$ & 2.0 & Lake Dgał Wielki & $1979-84$ & $\begin{array}{l}\text { Grabda- } \\
\text { Kazubska et al. } \\
1987\end{array}$ \\
\hline- & gills & $52.2^{3}$ & $1-16\left(3.4^{3}\right)$ & $1.79^{3}$ & Szczecin Lagoon & $1982-83$ & $\begin{array}{l}\text { Orecka-Grabda } \\
\text { and Wierzbicka } \\
1994\end{array}$ \\
\hline- & gills & 33.3 & $1-4\left(2.2^{3}\right)$ & 0.75 & Skolwiński Canal & $1982-83$ & $\begin{array}{l}\text { Orecka-Grabda } \\
\text { and Wierzbicka } \\
1994\end{array}$ \\
\hline- & - & 6 & - & - & Lake Śniardwy & 1989 & $\begin{array}{l}\text { Własow et al. } \\
1991\end{array}$ \\
\hline- & gills & 10.23 & $1-23(7.67)$ & 0.78 & Lake Łebsko & $2000-06$ & $\begin{array}{l}\text { Morozińska- } \\
\text { Gogol 2007, } \\
\text { Morozińska- } \\
\text { Gogol } 2011\end{array}$ \\
\hline- & - & 9 & $1-4(2.3)$ & $0.20^{3}$ & Vistula Lagoon & 2005 & $\begin{array}{l}\text { Rolbiecki and } \\
\text { Rokicki } 2006\end{array}$ \\
\hline A & gills & $100^{3,9}$ & $\left(3.0^{3}\right)$ & $1.50^{3}$ & $\begin{array}{l}\text { Lake } \\
\text { Żarnowieckie }\end{array}$ & 2007 & Rolbiecki 2011 \\
\hline A & gills & $10.0^{9}$ & $3(3.0)$ & 0.30 & $\begin{array}{l}\text { Lake } \\
\text { Choczewskie }\end{array}$ & $2008-15$ & This study \\
\hline A & gills & $20.0^{9}$ & $7(7.0)$ & 1.40 & River Parsęta & 2014-16 & This study \\
\hline A & gills & $20.0^{9}$ & $5(5.0)$ & 1.00 & Lake Jasień & 2015 & This study \\
\hline$A$ & gills & $28.6^{9}$ & $2-3(2.5)$ & 0.71 & Lake Sarbsko & 2016 & This study \\
\hline
\end{tabular}




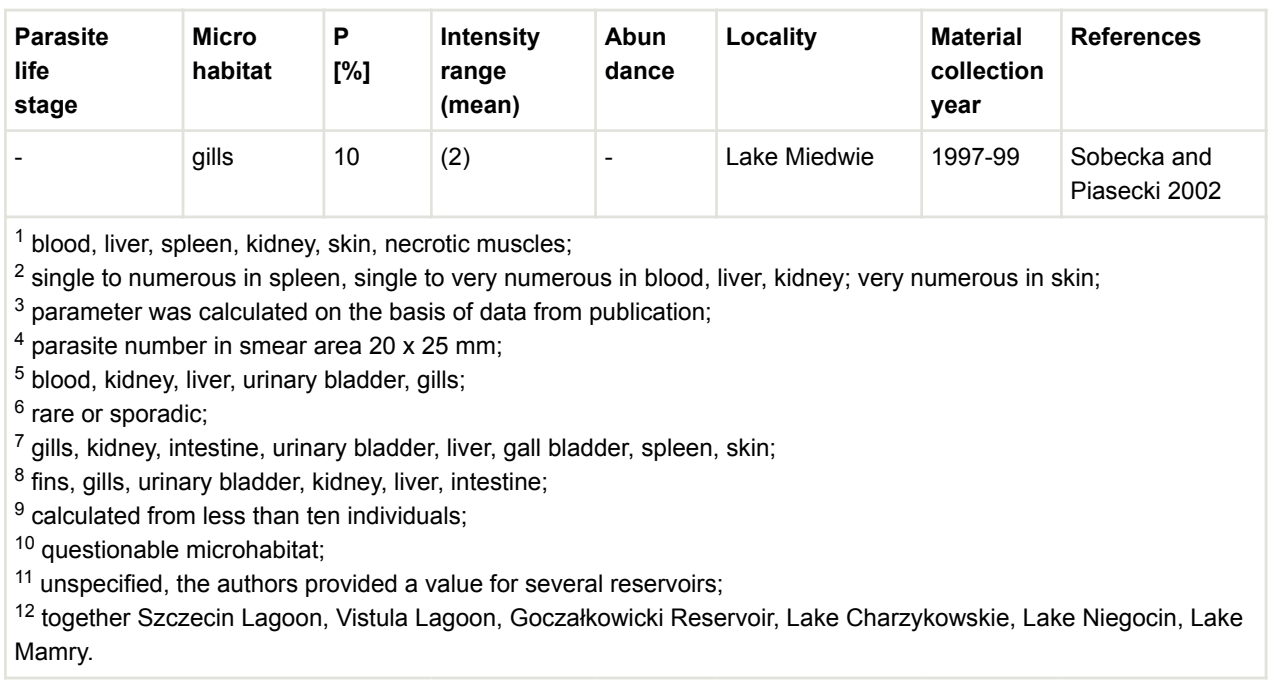

For comparison, the list of European eel parasites (data until 2009) from 30 countries in Europe and North Africa specifies 161 parasitic taxa (129 identified to species), of which 146 were metazoans and 15 were Protista (Jakob et al. 2016): Epieimeria anguillae and Eimeria anguillae, which were listed as two distinct species, are now considered to be the same taxon in the genus Eimeria, according to Benajiba et al. (1994). Similarly to the Polish study, digenetic trematodes (39 species) and nematodes (38 species) formed the most species-rich groups.

Twenty six parasite species, included in the present list, are also present in Jakob et al. (2016). However, that list does not include many parasite species and localities from Poland and does not reflect the actual distribution of the parasites. For example, Poland was not given as the area of occurrence for 23 parasite species (Trypanosoma granulosum, Apiosoma sp., Ichthyophthirius multifiliis, Trichodina jadranica, Trichodinella epizootica, Capriniana piscium, Rhabdospora thelohani, Henneguya psorospermica, Myxobolus portucalensis, Myxidium giardi, Zschokkella stettinensis, Ortholinea sphaerocapsularae, Bunodera luciopercae, Dactylogyrus sp., Triaenophorus nodulosus, Cystidicola farionis, Spinitectus inermis, Hysterothylacium aduncum, Paraquimperia tenerrima, Corynosoma strumosum, Echinorhynchus gadi, Echinorhynchus truttae and Paratenuisentis ambiguus) in Jakob et al. (2016). In addition, a number of groups and species, included in the present list, were absent from Jakob et al. (2016). For instance, the group of protists from Poland has now been expanded to include Capriniana piscium and Ortholinea sphaerocapsularae and a representative of Apiosoma without species identification. Rhabdospora thelohani is also mentioned; however, considerable controversy exists as to whether this species is indeed a representative of apicomplexan parasites or a host "rodlet cell" (Davies and Ball 1993). In addition, amongst the Metazoa, the new list has been enriched with the addition of Henneguya psorospermica, Cystidicola farionis and an unidentified Dactylogyrus species for Poland, as well as unidentified cestodes found by Sołtyńska (1964). The present list includes a number of new localities for eel parasites, previously unpublished (marked in Table 1 as "this study") 
In comparison, only nine species of parasites were recorded for European eels in Japan, as well as seven taxa identified at genus level and an unidentified Monogenea. However, these eels also included parasites, thus far unknown from the European $A$. anguilla (Cryptobia spp., Ichthyobodo spp., Gyrodactylus anguillae, Lernaea cyprinacea) (Nagasawa et al. 2007, Nagasawa and Hirotaka 2017). This confirms the possibility that regional differences may exist, not only with regard to the level of infection, but also in the composition and species diversity of the parasite fauna.

Within the parasitofauna of eel, the greatest repeatability between different distribution areas is exhibited by the parasites specific to the genus Anguilla (e.g. the nematode $A$. crassus, the cestodes Bothriocephalus claviceps and Proteocephalus macrocephalus or the trematode Deropristis inflata), but also certain widely-distributed species with large ranges of hosts, such as the trematode Diplostomum spathaceum s. I., the leech Piscicola geometra or the copepod Ergasilus sieboldi. It is in this area that the number of records of alien and invasive parasites increases for the European eel; for example, $A$. crassus, which was introduced to Europe in 1982 and recorded in Poland in 1988 (Koops and Hartmann 1989, Własow 1991, Bystydzieńska et al. 2005) or Pseudodactylogyrus anguilla and P. bini, recorded in Poland in 1995 by Dzika et al. (1995). It is also important to note the presence of a new, potentially invasive species, the acanthocephalan Paratenuisentis ambiguus, originally a parasite of Anguilla rostrata (Lesueur, 1817), which was first recorded in a European eel from Europe in 1980 and later in Poland by Morozińska-Gogol (2008).

The dispersal of parasites, their increased prevalence and level of infection are linked not only to the life history of eels and their migrations. Parasite infection in local eel populations can also transmitted through stocking material. For instance, protozoan Trichodina fultoni (100\% infection) was found in rearing glass eel imported to Poland from France in 1971 (Markiewicz and Migała 1980). In addition, the monogenean $P$. anguillae and the nematode $A$. crassus were found in eels originating from the stocking material for the Vistula Lagoon in 2006 (Rolbiecki et al. 2008). What is more, of the eels originating from farming facilities located in the Warmian-Masurian Voivodeship (northeast Poland) and studied in the period 2010-2014, 77.2\% were found to have Pseudodactylogyrus spp.; Trichodina spp. and I. multifiliis were typical parasites at the early rearing stage (Terech-Majewska et al. 2016).

With their resources already being considerably depleted and the growing number threats to eel populations, there has been a growing interest in their parasitic fauna; this growth has been accompanied by a greater need to carry out regular monitoring of parasitological threats, especially actual or potentially pathogenic species, including alien and invasive species. However, such data have to be constantly supplemented and verified with new records of parasites in different parts of distribution of this host. As such research would be complicated by the degree of data scatter, the best solution would be to create a webbased database, supplemented and coordinated by scientific centres from different countries. 


\section{References}

- Amin O (2013) Classification of the Acanthocephala . Folia Parasitologica 60 (4): 273-305. https://doi.org/10.14411/fp.2013.031

- Benajiba MH, Marques A, Lom J, Bouix G (1994) Ultrastructure and sporogony of Eimeria (syn. Epieimeria) anguillae (Apicomplexa) in the eel (Anguilla anguilla). Journal of Eukaryotic Microbiology 41 (3): 215-222. https://doi.org/10.1111/i. 1550-7408.1994.tb01500.x

- $\quad$ Bielecki A, Cichocka JM, Jeleń J, Światek P, Adamiak-Brud Z (2011) A checklist of leech species from Poland. Wiadomości Parazytologiczne 57 (1): 11-20.

- $\quad$ Bystydzieńska Z, Rolbiecki L, Rokicki J (2005) Helminth communities of European eels Anguilla anguilla (Linnaeus, 1758) from the Vistula Lagoon and Puck Bay, Poland. Wiadomości Parazytologiczne 51 (2): 145-150.

- Dabrowska Z (1970) Fish parasites of the Vistula River near Warszawa. Acta Parasitologica Polonica 27 (21): 189-193.

- Davies AJ, Ball SJ (1993) The biology of fish coccidia. Advances in Parasitology 32: 293-366. https://doi.org/10.1016/s0065-308x(08)60210-9

- Dekker W, van Os B, van Willigen J (1998) Minimal and maximal size of eel. Bulletin Français de la Pêche et de la Pisciculture 349: 195-197. https://doi.org/10.1051/kmae: 1998044

- $\quad$ Dekker W (2003) Status of the European eel stock and fisheries. In: Aida K, Tsukamoto K, Yamauchi K (Eds) Eel Biology. Springer Japan, Tokyo, 237-254 pp. https://doi.org/ 10.1007/978-4-431-65907-5 17

- Dzika E, Własow T, Gomułka P (1995) The first recorded case of the occurrence of two species of the genus Pseudodactylogyrus on the eel Anguilla anguilla (L.) in Poland. Acta Parasitologica 40 (4): 165-167.

- $\quad$ Dzika E (1999) Microhabitats of Pseudodactylogyrus anguillae and P. bini (Monogenea: Dactylogyridae) on the gills of large-size European eel Anguilla anguilla from Lake Gaj, Poland. Folia Parasitologica 46 (1): 33-36.

- $\quad$ Einszporn-Orecka T (1979) Flagellates Spironucleus anguillae sp. n. parasites of eel (Anguilla anguilla L.). Acta Protozoologica 18 (2): 237-241.

- Freyhof J, Kottelat M (2010) Anguilla anguilla. The IUCN Red List of Threatened Species 2010: e.T60344A12353683. www.iucnredlist.org/species/60344/12353683. Accessed on: 2020-3-06.

- Garbacik-Wesołowska A, Szkudlarek A, Sobecka E (1994) Nematode Anguillicola crassus in eels from the Szczecin Lagoon and Lake Łętowskie. Bulletin of the Sea Fisheries Institute 2 (132): 34-37.

- Georgieva S, Soldánová M, Pérez-del-Olmo A, Dangel D, Sitko J, Sures B, Kostadinova A (2013) Molecular prospecting for European Diplostomum (Digenea: Diplostomidae) reveals cryptic diversity. International Journal for Parasitology 43 (1): 57-72. https:// doi.org/10.1016/j.ijpara.2012.10.019

- $\quad$ Gibson DI, Jones A, Bray RA (2002) Keys to the Trematoda . Volume1. CAB International, Wallingford, 521 pp. https://doi.org/10.1079/9780851995472.0000

- $\quad$ Grabda E, Grabda J, Wierzbicki K (1961) Pasożyty i choroby ryb w jeziorze Wdzydze. Roczniki Nauk Rolniczych 93-D: 239-266. 
- $\quad$ Grabda J (1962) Pasożytnicze widłonogi (Copepoda parasitica) ryb Zalewu Wiślanego. Prace Morskiego Instytutu Rybackiego w Gdyni 11/A: 275-286.

- Grabda J (1967) Widłonogi pasożytnicze (Copepoda parasitica) i tarczenice (Branchiura). Katalog Fauny Polski. PWN, Warszawa, 27 pp.

- Grabda J (1971) Pasożyty kragłoustych i ryb (Parasiti Cyclostomatorum et Piscium). Katalog Fauny Pasożytniczej Polski. PWN, Warszawa - Wrocław, 304 pp.

- Grabda J (1972) Pasożytnicze widłonogi /Copepoda parasitica/ w przybałtyckich wodach Polski. Studia i Materiały Oceanologiczne 3: 219-225.

- Grabda-Kazubska B, Baturo-Warszawska B, Pojmańska T (1987) Dynamics of parasite infestation of fish in lakes Dgał Wielki and Warniak in connection with introduction of phytophagous species. Acta Parasitologica Polonica 32 (1): 1-27.

- Grawiński E (1994) Occurence of Anguillicola crassa nematode in eel (Anguilla anguilla) from Vistula Lagoon and Pomeranian lakes. 17th Congress of the Polish Parasitological Society, Gdynia, 15-17 September. Instytut Medycyny Morskiej i Tropikalnej. Biulety Metodyczno-Organizacyjny, 27, $55 \mathrm{pp}$.

- Hohenadler MAA, Nachev M, Thielen F, Taraschewski H, Grabner D, Sures B (2018) Pomphorhynchus laevis: An invasive species in the river Rhine? Biological Invasions 20 (1): 207-217. https://doi.org/10.1007/s10530-017-1527-9

- Jakob E, Walter T, Hanel R (2016) A checklist of the protozoan and metazoan parasites of European eel (Anguilla anguilla): checklist of Anguilla anguilla parasites. Journal of Applied Ichthyology 32 (4): 757-804. https://doi.org/10.1111/j.1439-0426.2009.01345.x

- Jarecka L (1959) On the life-cycle of Bothriocephalus claviceps (Goeze, 1782). Acta Parasitologica Polonica 7 (27): 527-533.

- Jeżewski W, Laskowski Z, Zdzitowiecki K (2007) Helminth fauna of fish from Kuc Lake. Wiadomości Parazytologiczne 53 (suppl.): 214.

- Jones A, Bray RA, Gibson DI (2005) Keys to the Trematoda . 2. CAB International, Wallingford, 745 pp. https://doi.org/10.1079/9780851995878.0000

- Kahlil LF, Jones A, Bray RA (1994) Keys to the cestode parasites of vertebrates. CAB International, Wallingford, $751 \mathrm{pp}$.

- $\quad$ Kirk RS (2003) The impact of Anguillicola crassus on European eels. Fisheries Management and Ecology 10 (6): 385-394. https://doi.org/10.1111/j.

1365-2400.2003.00355.x

- Koops H, Hartmann F (1989) Anguillicola-infestations in Germany and in German eel imports. Journal of Applied Ichthyology 5 (1): 41-45. https://doi.org/10.1111/j. 1439-0426.1989.tb00568.x

- Kozicka J (1959) Parasites of fishes of Drużno Lake. Acta Parasitologica Polonica 7 (1): 1-72.

- Kozikowska Z (1957) Skorupiaki pasożytnicze (Crustacea parasitica) Polski. Część I. Pasożyty ryb wód ujściowych Odry. Zoologica Poloniae 8 (2-3): 217-270.

- Kozikowska Z (1965) Crustacés, parasites des poissons de la Pologne III. Resultats des explorations sur les poissons de la cote méridionale de la mer Baltique. Polskie Archiwum Hydrobiologii 13 (26) (1): 97-104.

- Lefebvre F, Contournet P, Crivelli AJ (2002) The health state of the eel swimbladder as a measure of parasite pressure by Anguillicola crassus . Parasitology 124 (4): 457-463. https://doi.org/10.1017/s0031182001001378

- Lom J, Dyková I (1992) Protozoan parasites of fishes (Developments in aquaculture and fisheries science). Elsevier, Amsterdam, $316 \mathrm{pp}$. 
- $\quad$ Markiewicz F, Migała K (1980) Trichodinid invasion (Peritricha, Urceolariidae) on young eels (Anguilla anguilla L.) grown in aquaria. Acta Hydrobiologica 22 (2): 229-236.

- Markowski S (1933) Die Eingeweidewürmer der Fische des polnischen Balticums (Trematoda, Cestoda, Nematoda, Acanthocephala). Archives d'Hydrobiologie et d'Ichthyologie 7: 1-58.

- Molnár K, Baska F, Csaba G, Glávits R, Székely C (1993) Pathological and histopathological studies of the swimbladder of eels Anguilla anguilla infected by Anguillicola crassus (Nematoda: Dracunculoidea). Diseases of Aquatic Organisms 15: 41-50. https://doi.org/10.3354/dao015041

- Moravec F (1992) Spreading of the nematode Anguillicola crassus (Dracunculoidea) among eel populations in Europe. Folia Parasitologica 39 (3): 247-248.

- Moravec F (2006) Dracunculoid and anguillicoloid nematodes parasitic in vertebrates. Academia, Prague, $634 \mathrm{pp}$.

- Moravec F (2013) Parasitic nematodes of freshwater fishes of Europe. Academia, Prague, $601 \mathrm{pp}$.

- Moriarty C, Dekker W (1997) Management of the European eal. Marine Institute, 110 pp. URL: http://hdl.handle.net/10793/197

- Morozińska-Gogol J (2005) Occurrence of the asiatic nematode Anguillicola crassus in European eel from the Łebsko Lagoon (Central Coast, Poland). Oceanological and Hydrobiological Studies 34 (suppl. 1): 113-119.

- Morozińska-Gogol J (2007) Metazoan parasites of fish from the Łebsko Lagoon (Central Coast, Poland). Baltic Costal Zone 11: 51-58.

- Morozińska-Gogol J (2008) The first record of Paratenuisentis ambiguus (Acanthocephala, Tenuisentidae) in Poland. Oceanologia 50 (2): 275-279.

- Morozińska-Gogol J (2009) Alien species of fish parasites in the coastal lakes and lagoons of the southern Baltic. Oceanologia 51 (1): 105-115. https://doi.org/10.5697/oc. $\underline{51-1.105}$

- Morozińska-Gogol J (2011) Pasożytnicze Metazoa ryb z jeziora Łebsko. Wydawnictwo Naukowe Akademii Pomorskiej w Słupsku, Słupsk, 176 pp.

- Münderle M, Taraschewski H, Klar B, Chang C, Shiao J, Shen K, He J, Lin S, Tzeng W (2006) Occurrence of Anguillicola crassus (Nematoda: Dracunculoidea) in Japanese eels Anguilla japonica from a river and an aquaculture unit in SW Taiwan. Diseases of Aquatic Organisms 71: 101-108. https://doi.org/10.3354/dao071101

- $\quad$ Nadler SA, D'Amelio S, Dailey MD, Paggi L, Siu S, Sakanari JA (2005) Molecular phylogenetics and diagnosis of Anisakis, Pseudoterranova, and Contracaecum from northern Pacific marine mammals. Journal of Parasitology 91 (6): 1413-1429. https:// doi.org/10.1645/GE-522R.1

- Nagasawa K, Umino T, Mizuno K (2007) A checklist of the parasites of eels (Anguilla spp.) (Anguilliformes: Anguillidae) in Japan (1915-2007). Journal of the Graduate School of Biosphere Science 46: 91-122.

- Nagasawa K, Hirotaka K (2017) A revised and updated checklist of the parasites of eels (Anguilla spp.) (Anguilliformes: Anguillidae) in Japan (1915-2017). Biosphere Science 53: 33-69. https://doi.org/10.15027/44658

- Niewiadomska K (2003) Pasożyty ryb Polski (klucze do oznaczania). Przywry Digenea. Polskie Towarzystwo Parazytologiczne, Warszawa, 169 pp.

- $\quad$ Orecka-Grabda T (1986) Hematological, clinical and anatomical and anatomical pathology of the european eel (Anguilla anguilla (L.)) from polluted waters of 
Northwestern Poland. Acta Ichthyologica et Piscatoria 16 (1): 107-127. https://doi.org/ 10.3750/AIP1985.16.1.09

- $\quad$ Orecka-Grabda T, Wierzbicka J (1994) Metazoan parasites of the eel, Anguilla anguilla (L.) in the Szczecin Lagoon and River Odra mouth area. Acta Ichthyologica et Piscatoria 24 (2): 13-19. https://doi.org/10.3750/aip1994.24.2.02

- $\quad$ Orecka-Grabda T, Wierzbicka J (1996) Observations on Trypanosoma granulosum Laveran et Mesnil, 1902 (Protozoa, Kinetoplastida), a blood parasite of eel, Anguilla anguilla (L.). Acta Ichthyologica et Piscatoria 26 (1): 39-47. https://doi.org/10.3750/ aip1996.26.1.04

- $\quad$ Orecka T, Pilecka-Rapacz M, Rząd I (1995) Pasożyty - azjatyckie nicienie - przyczyną wyniszczającego schorzenia węgorzy. Przegląd Rybacki 2: 50-55.

- $\quad$ Pilecka-Rapacz M (2001) Występowanie Anguillicola crassus u występującego węgorza rzek pomorza zachodniego. Roczniki Naukowe PZW 14: 115-123.

- $\quad$ Pilecka-Rapacz M, Sobecka E (2004) Nematodes of the intestine and swimbladder of the European eel Anguilla anguilla (L.) ascending Pomeranian rivers. Wiadomości Parazytologiczne 50 (1): 19-28.

- Pojmańska T, Niewiadomska K, Okulewicz A (2007) Pasożytnicze helminty Polski. Gatunki, żywiciele, białe plamy. Polskie Towarzystwo Parazytologiczne, Warszawa, 360 pp.

- $\quad$ Popielarczyk R, Robak S, Siwicki K (2012) Infection of European eel, Anguilla anguilla (L.), with the nematode Anguillicoloides crassus (Kuwahara, Niimi et Itagaki, 1974) in Polish waters. Polish Journal of Veterinary Sciences 15 (2): 253-257. https://doi.org/ 10.2478/v10181-011-0142-4

- Popiołek M (2016) Pasożyty ryb Polski (klucze do oznaczania). Kolcogłowy Acanthocepha. Polskie Towarzystwo Parazytologiczne, Warszawa, 79 pp.

- $\quad$ Rokicki J (1975) Helminth fauna of fishes of the Gdańsk Bay (Baltic Sea). Acta Parasitologica Polonica 23 (2): 37-84.

- $\quad$ Rolbiecki L, Grawiński E, Rokicki J (1996) The occurence of nematod Anguillicola crassus Kuwahara, Niimi et Itagaki 1974 in the swimbladder of eel (Anguilla anguilla L.). Land-Ocean Interactions in the Coastal Zone, Second International Symposium on: Functioning of Coastal Ecosystems in Various Geographical Regions, Sopot, 5-7 September. 55-56 pp.

- $\quad$ Rolbiecki L, Rokicki J, Wojtkiewicz D (2000) The first record of the nematode Anguillicola crassus (Nematoda: Dracunculoidea) in eel of the Gulf of Gdańsk (Poland). Oceanological Studies 29 (2): 75-81.

- Rolbiecki L, Rokicki J (2005) Anguillicola crassus - an alien nematode species from the swim bladders of eel (Anguilla anguilla) in the Polish zone of the Southern Baltic and in the waters of Northern Poland. Oceanological and Hydrobiological Studies 35 (suppl. 1): 121-136.

- Rolbiecki L, Rokicki J (2006) Parasite fauna of the eel, Anguilla anguilla (Linnaeus, 1758), from the Polish part of the Vistula Lagoon. Wiadomości Parazytologiczne 52 (2): 115-119.

- Rolbiecki L (2008) New data on the biology of the introduced exotic nematode Anguillicola crassus Kuwahara, Niimi et Itagaki, 1974 in the eel Anguilla anguilla in Lake Wdzydze (Polish waters). Oceanological and Hydrobiological Studies 37 (3): 37-48. 
- $\quad$ Rolbiecki L, Bartel R, Rokicki J (2008) The nematode parasite, Anguillicola crassus Kuwahara, Niimi et Itagaki, and the monogenean gill parasite, Pseudodactylogyrus anguillae (Yin et Sproston), in eel, Anguilla anguilla (L.), fry. Archives of Polish Fisheries 16 (2): 221-226. https://doi.org/10.2478/s10086-008-0019-z

- Rolbiecki L (2011) Nowe dane na temat rozprzestrzenienia inwazyjnego nicienia Anguillicoloides crassus (Anguillicolidae) u węgorzy na terenie Polski. Komunikaty Rybackie 4 (123): 9-13.

- Rząd I, Pilecka-Rapacz M (2001) Wpływ Anguillicola crassus na wskaźniki krwi obwodowej węgorzy Anguilla anguilla L. z Zalewu Szczecińskiego. Acta Biologica 8: 45-54.

- $\quad$ Rząd I, Pilecka-Rapacz M (2002) Zmiany hematologiczne u węgorza europejskiego Anguilla anguilla zarażonego przez Anguillicola crassus (Kuwahara, Niimi, Itagaki, 1974). Acta Biologica 9: 45-54.

- Rząd I, Pilecka-Rapacz M, Sobecka E (2007) Characteristics of the peripheral blood of the Anguillicola crassus (Kuwahara, Niimi et Itagaki, 1974) and Trypanosoma granulosum Laveran et Mesnil, 1902 infested eel (Anguilla anguilla L.) ascending the River Rega (north-western Poland). Wiadomości Parazytologiczne 53 (4): 319-324.

- $\quad$ Seyda M (1973) Parasites of eel Anguilla anguilla (L.) from the Szczecin firth and adjacent waters. Acta Ichthyologica et Piscatoria 3 (2): 67-76. https://doi.org/10.3750/ AIP1973.03.2.04

- Sitjà-Bobadilla A, Alvarez-Pellitero P (1994) Revised classification and key species of the genus Sphaerospora Davies, 1917 (Protozoa: Myxosporea). Research and Reviews in Parasitology 54 (2): 67-80.

- $\quad$ Sitowski L (1937) O masowym występowaniu pijawki Cystobranchus respirans Troschel w Dunajcu i jego dopływach. Przegląd Rybacki 10 (5): 185-187.

- Sobecka E, Piasecki W (2002) Parasite fauna of selected fish species of Lake Miedwie. Wiadomości Parazytologiczne 48 (2): 207-215.

- Sobecka E, Pilecka-Rapacz M (2003) Pseudodactylogyrus anguillae (Yin et Sproston, 1948) Gussev, 1965 and P. bini (Kikuchi, 1929) Gussev, 1965 (Monogenea:

Pseudodactylogyridae) on gills of European eel, Anguilla anguilla (Linnaeus, 1758) ascending rivers of the Pomerani. Acta Ichthyologica et Piscatoria 33 (2): 137-143.

https://doi.org/10.3750/aip2003.33.2.03

- Sołtyńska M (1964) Fish tapeworms in Puck Bay (South Baltic). Acta Parasitologica Polonica 12 (3): 13-26.

- S S S Sakulová M, Perrot-Minnot M, Neuhaus B (2011) Resurrection of Pomphorhynchus tereticollis (Rudolphi, 1809) (Acanthocephala: Pomphorhynchidae) based on new morphological and molecular data. Helminthologia 48 (4): 268-277. https://doi.org/ 10.2478/s11687-011-0038-y

- $\quad$ Stone R (2003) Freshwater eels are slip-sliding away. Science 302 (5643): 221-222. https://doi.org/10.1126/science.302.5643.221

- Styczyńska E (1958) Acanthocephala of the biocoenosis of Drużno Lake [Parasitofauna of the biocoenosis of Drużno Lake - part VI]. Acta Parasitologica Polonica 6 (6): 195-211.

- Sulgostowska T (1993) Parasites of eel, Anguilla anguilla (L.) from south-east Baltic Sea (Poland). Acta Parasitologica 38 (2): 82-84.

- Svobodová Z, Kolářová J, Dyková I, Hamáčková J, Kouřil J (2009) Infection by Capriniana piscium (Buetschli, 1889) Jankovski, 1973, a cause of rainbow trout 
(Oncorhynchus mykiss) kill. Bulletin- European Association of Fish Pathologists 29 (3): 92-97.

- $\quad$ Szidat L (1944) Weitere Untersuchungen über die Trematodenfauna einheimischer Süsswasserfische. Zeitschrift für Parasitenkunde 13 (2): 183-214. https://doi.org/ $10.1007 / \mathrm{bf02122205}$

- $\quad$ Taraschewski H, Moravec F, Lamah T, Anders K (1987) Distribution and morphology of two helminths recently introduced into European eel populations: Anguillicola crassus (Nematoda, Dracunculoidea) and Paratenuisentis ambiguus (Acanthocephala, Tenuisentidae). Diseases of Aquatic Organisms 3: 167-176. https://doi.org/10.3354/ dao003167

- Terech-Majewska E, Bernad A, Robak S, Pajdak J, Schulz P, Siwicki AK, Szweda W (2016) Czynniki bakteryjne i pasożytnicze diagnozowane u węgorza europejskiego w Polsce w latach 2010-2014 w warunkach podchowu kontrolowanego. Medycyna Weterynaryjna 72 (10): 647-651. https://doi.org/10.21521/mw.5568

- van Ginneken VT, Maes G (2005) The European eel (Anguilla anguilla, Linnaeus), its lifecycle, evolution and reproduction: a literature review. Reviews in Fish Biology and Fisheries 15 (4): 367-398. https://doi.org/10.1007/s11160-006-0005-8

- Wegener G (1909) Die Ektoparasiten der Fische Ostpreußens. Schriften der Physikalisch-ökonomischen Gesellschaft zu Königsberg 50: 195-286.

- Wierzbicka J (1986a) Sphaerospora anguillae sp. n. (Myxospora, Bivalvulida), a parasite of eel, Anguilla anguilla (L.). Acta Protozoologica 25 (1): 119-122.

- Wierzbicka J (1986b) Sphaerospora sphaerocapsularae sp. n. (Myxospora, Bivalvulida) a parasite of eel, Anguilla anguilla (L.). Acta Protozoologica 25 (3): 355-358.

- Wierzbicka J, Einszporn-Orecka T (1986) Flagellates Spironucleus mobilis sp. n. in eel Anguilla anguilla (L.). Acta Protozoologica 25 (1): 75-80.

- Wierzbicka J (1987) Zschokkella stettinensis sp. n. (Myxospora, Bivalvulida) - a parasite of eel, Anguilla anguilla (L.). Acta Protozoologica 26 (1): 79-82.

- Wierzbicka J (1994) Revision of the Sphaerospora Thélohan, 1892 (Myxosporea) protozoans parasitising the eel, Anguilla anguilla (L.). Acta Ichthyologica et Piscatoria 24 (2): 21-24. https://doi.org/10.3750/aip1994.24.2.03

- Wierzbicka J, Orecka-Grabda T (1994) Protozoans parasitic on Anguilla anguilla (L.) from the Szczecin Lagoon and River Odra mouth. Acta Ichthyologica et Piscatoria 24 (2): 3-11. https://doi.org/10.3750/AIP1994.24.2.01

- Wierzbicka J, Orecka-Grabda T (1996) Myxobolus portucalensis Saraiva \& Molnar, 1990 in various organs of eel, Anguilla anguilla (L.). Acta Ichthyologica et Piscatoria 26 (2): 95-101. https://doi.org/10.3750/aip1996.26.2.06

- Własow T (1991) Azjatycki nicień Anguillicola spp. w pęcherzu pławnym węgorza europejskiego Anguilla anguilla L. Komunikaty Rybackie 3: 21-22.

- Własow T, Kujawa R, Bernard A, Zielonka M (1991) Występowanie pasożytów u Anguilla anguilla (L.) na podstawie importowanego materiału obsadowego i węgorzy żerujących w wodach polskich. 16. Zjazd Polskiego Towarzystwa Parazytologicznego, Poznań, 12-13 Września. 35 pp.

- WoRMS Editorial Board (2020) World Register of Marine Species. http:// www.marinespecies.org. Accessed on: 2020-3-09.

- Zaddach EG (1844) Synopseos Crustaceorum Prussicorum Prodromus. Dissertation Zoologica. Regiomonti, 39 pp. https://doi.org/10.5962/bhl.title.11422 


\section{Supplementary material}

Suppl. material 1: GPS coordinates of collection sites doi

Authors: Joanna Dzido, Leszek Rolbiecki, Joanna N. Izdebska, Rafał Bednarek Data type: Table

Download file $(25.59 \mathrm{~kb})$ 